\title{
An Investigation into the Tunnel-Soil-Pipeline Interaction by In Situ Measured Settlements of the Pipelines
}

\author{
Xinggao Li $\mathbb{D},{ }^{1}$ Ting Wang, ${ }^{2}$ and Yi Yang ${ }^{1}$ \\ ${ }^{1}$ Key Laboratory of Urban Underground Engineering of Ministry of Education, Beijing Jiaotong University, Beijing 100044, China \\ ${ }^{2}$ Beijing Metro Construction Administration Corporation Ltd., Beijing 100068, China \\ Correspondence should be addressed to Xinggao Li; lxg_njtu@163.com
}

Received 28 July 2020; Revised 28 August 2020; Accepted 18 September 2020; Published 1 October 2020

Academic Editor: Peixin Shi

Copyright (c) 2020 Xinggao Li et al. This is an open access article distributed under the Creative Commons Attribution License, which permits unrestricted use, distribution, and reproduction in any medium, provided the original work is properly cited.

Response of buried pipeline to tunnelling is of great concern in the subway construction. It is of paramount importance but difficult to estimate the influence of tunnelling on adjacent pipelines because of the complex tunnel-soil-pipeline interaction. The technique of in situ measured settlements of buried pipelines remains the standard approach for understanding this interaction and addressing the issue. The Huangzhuang station of the Beijing Subway is located in a densely populated area, with many buried pipelines in the close proximity; the shallow tunnelling method of pile-beam-arch (PBA method) was used to excavate the station tunnels; the shallow tunnelling of the station tunnels inevitably causes settlements of the ground surface and the buried pipelines. Direct monitoring of the pipelines by digging holes from the ground surface was performed during the station tunnel construction. In situ measured settlements of the ground surface and the buried pipelines caused by the subway construction were obtained. It is observed from the obtained results that the pipeline settlement development can be divided into four stages that are associated with different construction periods of the PBA method. Sharp increases in the pipeline settlement occurred in the specific stages (stages 2 and 4). It is concluded from comparisons between the pipeline settlement and the ground surface settlement that separation between steel or cast iron pipelines and the soil beneath occurs due to the tunnel construction. And the pipeline settlement is smaller than that of the ground surface. This finding has the practical implication that the ground surface can be monitored instead of the buried pipeline. Using this indirect pipeline monitoring, the pipeline safety can be conservatively evaluated. This study is an example for evaluating the shallow tunnelling-induced effects on adjacent buried pipelines and understanding the tunnel-soil-pipeline interaction under similar conditions.

\section{Introduction}

In recent years, the shallow tunnelling method has been widely used in subway construction in soft ground in China [1]. And the shallow tunnelling method of pile-beam-arch (PBA method) has been widely applied in the excavation of subway stations in Beijing [2]. Subway tunnel construction inevitably induces ground movements, which, if uncontrolled, can cause excessive deformations and damage to existing buried pipelines. Such situations are very common, particularly when building tunnels in densely populated areas. Thus, precautions are required to reduce the relevant risks and possible adverse effects on nearby buried pipelines, and response of the buried pipelines to neighbouring tunnelling is of great concern.
The safety of buried pipelines affected by adjacent tunnelling activities has been a main concern in underground space utilization, and many relevant studies have been conducted in recent decades using theoretical analysis and/or model testing. The green field displacement profiles determined using the Winkler-type models were formulated to address this problem (e.g., Attewell et al. [3], Bracegirdle et al. [4], Wang et al. [5], Yu et al. [6], and Ieronymaki and Whitttle [7]). Furthermore, more complex elastic or elastoplastic continuum solutions were given for the problem of tunnelling effects on existing pipelines (e.g., Klar et al. [8-10] and Vorster et al. [11]). A series of centrifuge tests were undertaken to understand the soil-pipe interaction and investigate effects of tunnelling on buried pipelines (e.g., 
Vorster et al. [12], Marshall et al. [13], and Shi et al. [14, 15]). Capable of analysing problems over complicated domains and approximating the complex tunnel-soil-pipeline interaction, numerical methods (such as the finite element method and the finite difference method) were widely used to model tunnelling effect on buried pipelines (e.g., Hunter [16], Wang et al. [17], Zhang et al. [18, 19], Z. Zhang and M. Zhang [20], Wham et al. [21], Shi et al. [22], and Shi et al. [23]). Analytical solution of pipelines subjected to differential ground movement that were normally used by engineers in design office as an initial design step was presented (e.g., Trifonov and Cherniy [24], Karamitros et al. [25], Kouretzis et al. [26], Saiyar et al. [27], Ni and Mangalathu [28], and Ni et al. [29]). To better understand the tunnel-soilpipe interaction, both centrifuge tests and numerical modelling were performed to investigate the effects of tunnelling on existing buried pipelines (e.g., Ma et al. [30]). Moreover, Son [31] developed a simplified numerical approach to investigate the effects of microtunnelling on buried pipelines parametrically and concluded that the response of buried pipes to microtunnelling-induced ground settlements highly depends on the soil-pipe interaction, including the separation and slippage of pipe from soil, along with the effects of the investigated parameters (pipe stiffness, ground loss, and pipe location). Zhang et al. [32] developed a fuzzy Bayesian network-based approach for safety risk analysis of tunnel-induced pipeline damage and presented a case concerning the safety analysis of underground buried pipelines adjacent to the construction of the Wuhan Yangtze River Tunnel to demonstrate the feasibility of the proposed approach and its application potential.

However, the pipeline is assumed to follow the estimated green field settlement profile without taking account of the pipeline stiffness, resulting in an overestimation of the bending moments and even mistakes in some cases. Beamon-spring analysis uses empirical soil springs to simplify the interaction between the pipe and the soil. However, all empirical soil reaction models were developed for rigid pipes, and calculations using empirical springs can result in very conservative estimation for flexible pipes. In addition, empirical springs can provide reasonable calculations for pipelines subjected to ground deformation in the horizontal plane, but not in the vertical plane. When facing the problem of the settlement of buried pipeline caused by subway station construction, model tests can only be used to study limited interaction features and sometimes are not realistic because of the lack of accounting of some basic factors such as pipeline age and joint. And it is almost impossible to completely duplicate the complicated excavation process of a subway station in the model test. The numerical methods can rigorously consider the tunnel-soil-pipe interaction and the excavation process [33]. However, due to the difficulty in the selection of the model parameters and the constitutive equations of soils, a numerical model needs to be compared with the true behaviours of pipelines or model test results and calibrated before credible calculation results can be obtained. In particular, the physical and mechanical parameters of a buried pipeline vary with its service time; such parameters are difficult to model in numerical methods.
Field observations remain the more reliable approach for understanding the interaction behaviour between buried pipelines and construction of tunnels.

In situ measured settlements of the buried pipelines caused by the tunnels construction of the Beijing subway Huangzhuang station are studied herein. The Huangzhuang station is located at a densely built area. At the construction site of the station, there exist many buried pipelines (e.g., for water, sewage, and gas transportations). Although most pipelines were dismantled and moved before the station construction, a few pipelines remained in place unchanged. The station tunnel construction would inevitably cause settlements of the above existing pipelines. The pipeline settlements and the ground surface settlement were monitored during the tunnel construction, thereby guaranteeing an information-oriented construction. The subway station tunnel construction was completed as scheduled and without any interruption of the operation of the buried pipelines.

\section{Huangzhuang Station Tunnel Construction}

As shown in Figure 1, Huangzhuang station, situated at the busy intersection of Zhongguancun Street and Zhichulu Street, is a transfer station between Line 10 and Line 4 of the Beijing subway. The Line 10 part of the station, $156.9 \mathrm{~m}$ in length, is a triple-arch-double-deck tunnel with the excavation size of $26.06 \mathrm{~m} \times 19.07 \mathrm{~m}$ and the cover depth to the middle crown of $5.7-6.5 \mathrm{~m}$. The Line 4 part of the station, $216.6 \mathrm{~m}$ in length, is also a triple-arch-double-deck tunnel on the two sides with the excavation size of $26.42 \mathrm{~m} \times 16.844 \mathrm{~m}$ and the cover depth to the middle crown of $7.3-7.5 \mathrm{~m}$; however, the middle part is a triple-arch-single-deck tunnel (the transfer section) with the size of $24.06 \mathrm{~m} \times 11.45 \mathrm{~m}$ and the cover depth of 12.5-12.6 m.

Fifty-six pipelines were identified near the station, thirty of which were in the construction influence area. The excavation area is mainly in silty clay, silt and silty clay laminae, gravel and medium-coarse sand laminae, fine sand and embedded gravel, and medium-coarse sand and gravel embedded in clayey silt, as shown in Figure 1. For physical and mechanical properties of the involved typical soils, refer Table 1. Because the ground water is approximately $14.5 \mathrm{~m}$ underneath the surface, dewatering was necessary before and during the construction.

On account of the heavy surface traffic, a shallow tunnelling method, instead of the cut-and-cover approach, was used to excavate the station main body. The shallow tunnelling method adopted for construction of the double-deck tunnels of the station was termed as the PBA method. This method has been used in the past and is currently being widely used to construct station tunnels in Beijing and other cities of China. As illustrated in Figure 2, the main steps of the construction method used are as follows: (1) forepoling and grouting were used to reinforce ground, the four drifts were excavated with a sequence excavation method, and the initial support consisted of shotcrete and lattice girders; (2) the side piles were driven into ground, the middle steel columns were installed from within the top drifts, and the 


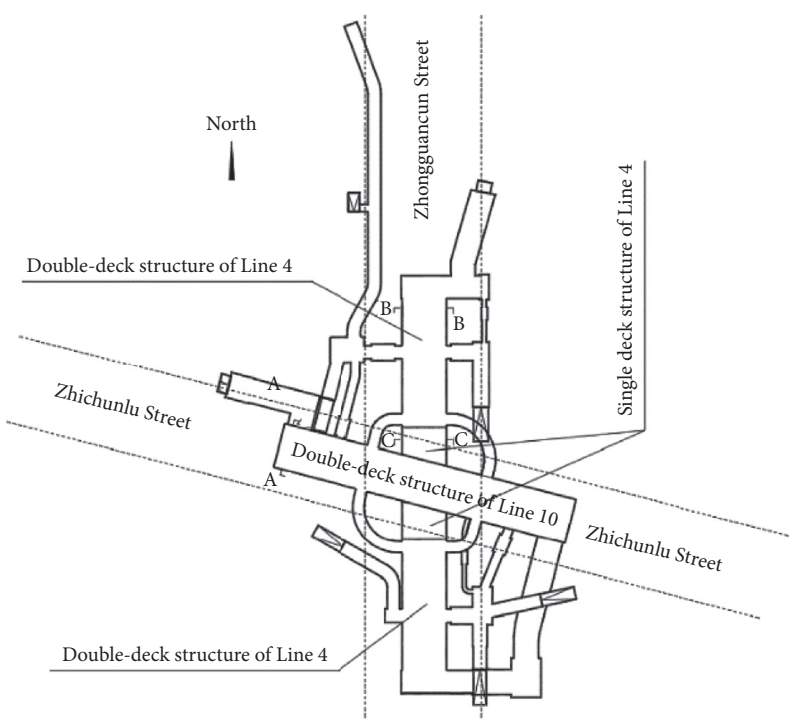

(a)
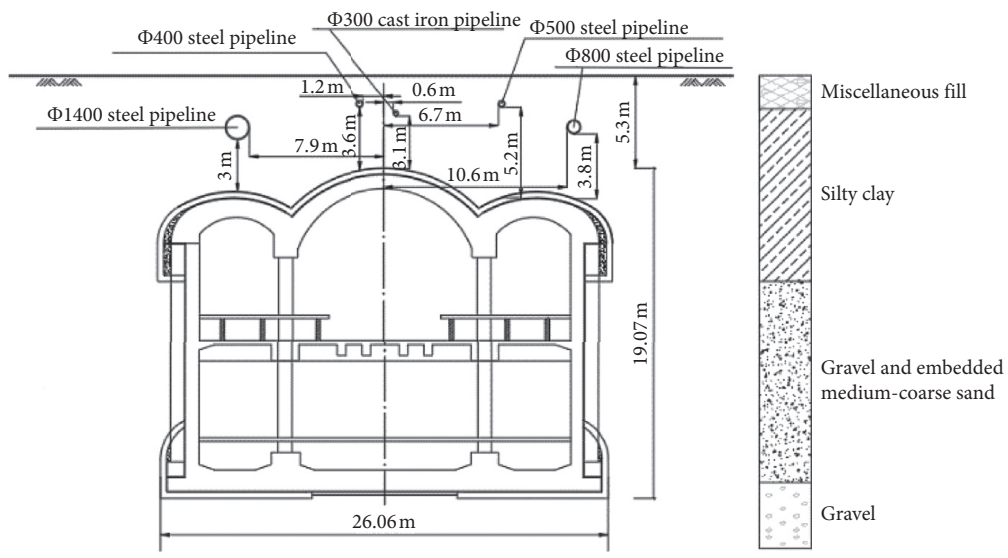

(b)
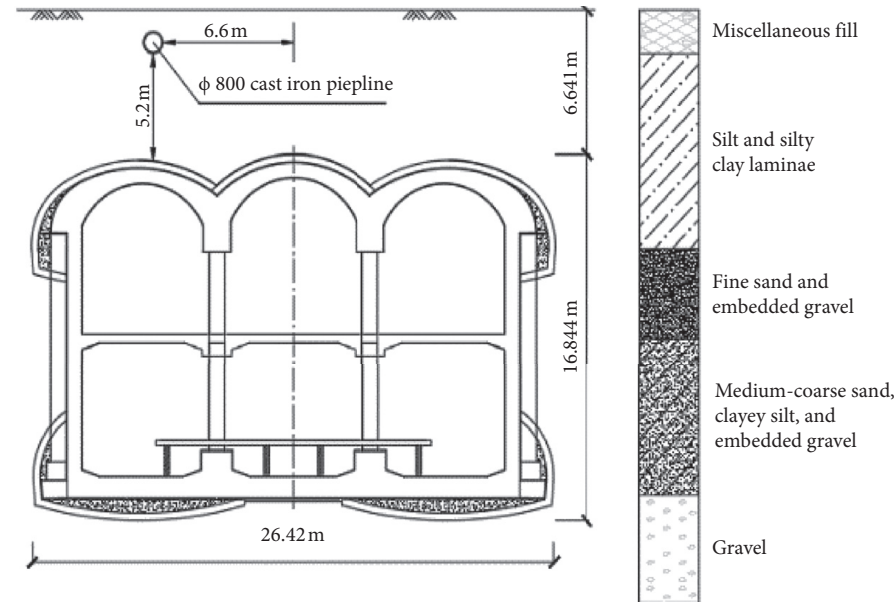

(c)

FIgUre 1: Continued. 


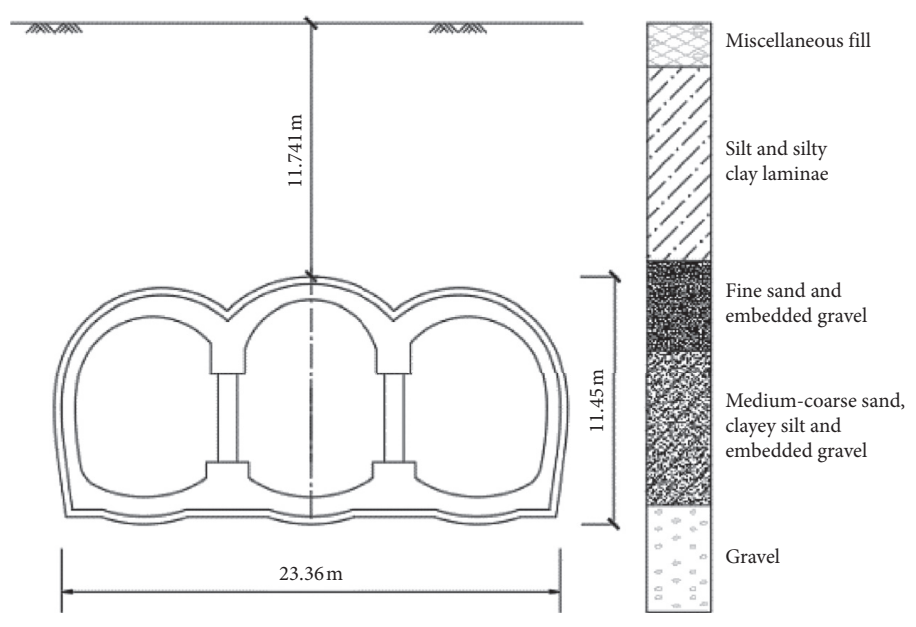

(d)

FIgURE 1: Layout of Huangzhuang station and the ground profile: (a) plane view; (b) section A-A; (c) section B-B; (d) section C-C.

TABLe 1: Physical and mechanical properties of the soils.

\begin{tabular}{|c|c|c|c|c|c|}
\hline Items & $\begin{array}{c}\text { Elastic modulus } \\
(\mathrm{MPa})\end{array}$ & Poisson's ratio & $\begin{array}{c}\text { Cohesion } \\
(\mathrm{kPa})\end{array}$ & $\begin{array}{c}\text { Friction angle } \\
\left({ }^{\circ}\right)\end{array}$ & $\begin{array}{c}\text { Density } \\
\left(\mathrm{kg} \cdot \mathrm{m}^{-3}\right)\end{array}$ \\
\hline Miscellaneous fill & 7 & 0.32 & 20 & 14 & 1800 \\
\hline Silty clay & 9 & 0.32 & 30 & 20 & 1900 \\
\hline Gravel and embedded medium-coarse sand & 55 & 0.28 & 5 & 40 & 2150 \\
\hline Gravel & 67 & 0.23 & 1 & 44 & 2150 \\
\hline
\end{tabular}
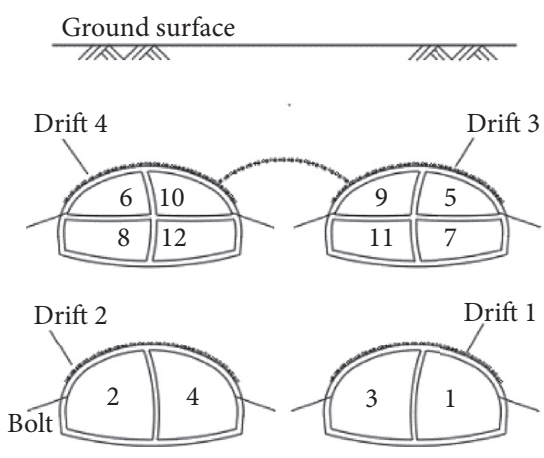

(a)

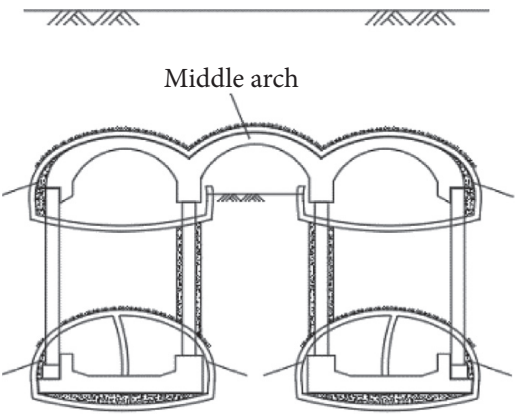

(d)

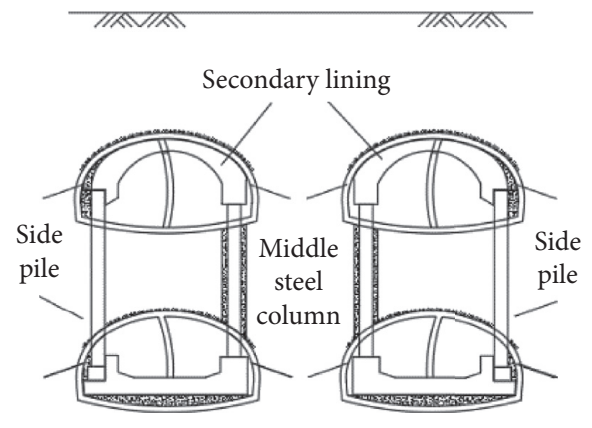

(b)

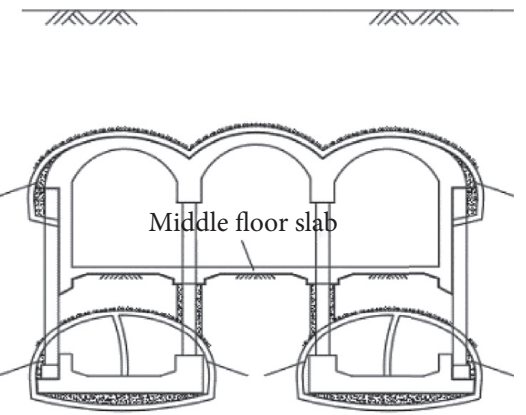

(e)

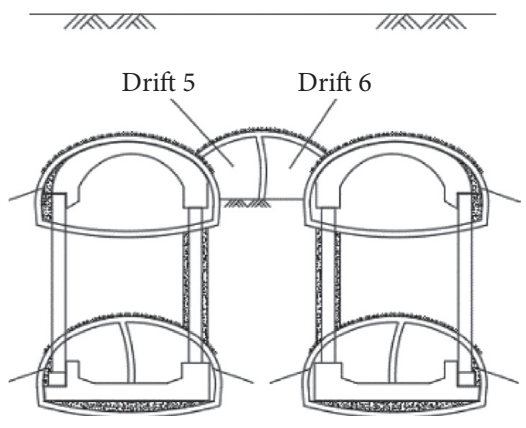

(c)
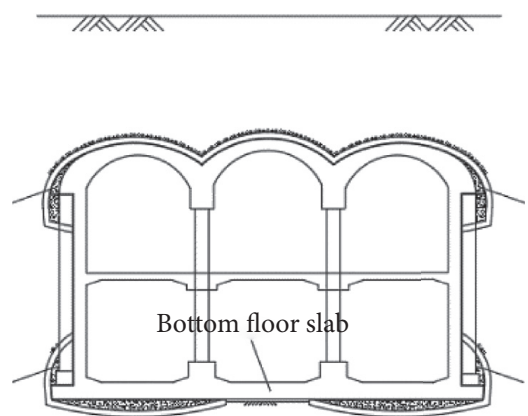

(f)

Figure 2: Main construction steps of the PBA method: (a) step 1; (b) step 2; (c) step 3; (d) step 4; (e) step 5; (f) step 6. 
secondary linings were cast; (3) ground reinforcement was implemented with forepoling and grouting, the drift 5 and drift 6 were excavated, and the initial support composed of shotcrete and lattice girders was employed; (4) the central diaphragm was demolished, and the secondary lining of the middle arch was cast; (5) the upper part was excavated using a top-down method with shotcrete primary lining and casting secondary lining, and the middle floor slab was built with concrete casting; (6) the remaining part was excavated, and the bottom floor slab was constructed with in situ concrete lining.

\section{Pipeline Monitoring}

3.1. Monitoring Scheme. The geological and hydrogeological conditions pose serious risks to the shallow tunnelling work, possibly causing large settlements of the pipelines. Thus, mastering the settlement development in the construction was of vital importance for the pipeline safety. To guide the information-oriented construction process, monitoring of the buried pipelines in terms of settlements was performed during the construction. Diversion of most of the pipelines was performed in advance of the tunnelling works. Six pipelines were chosen, after deliberate consideration, to be monitored: four steel pipelines ( $\phi 1400$ and $\phi 800$ for water supply and $\phi 400$ and $\phi 500$ for gas transportation) and one cast iron pipeline ( $\phi$ 300 for water supply) in the longitudinal direction above Line 10 and one $\phi 800$ cast iron water pipeline in the longitudinal direction above Line 4, as presented in Figure 3. The profiles of the six pipelines are given in Figure 1. For more detailed information of the monitored pipelines, refer Table 2.

For the pipeline lying below the road surface, a hole with a diameter of $800 \mathrm{~mm}$ was dug on the pipeline to directly monitor settlement of the pipeline, as illustrated in Figure 4(a), and the pipeline surface was exposed, as shown in Figure 4(b). A $\phi 50$ steel pipe was placed into the hole and set on the pipeline, and the space in-between the hole and the pipe was filled with sand. A $\phi 25$ steel bar was also placed into the $\phi 50$ steel pipe, and the pipeline settlement was monitored by measuring settlement of the steel bar top end, as shown in Figure 4(c). The $\phi 50$ steel pipe was tightly fixed on the monitored pipeline with adhesive, which guaranteed that the $\phi 50$ steel bar was vertical throughout the monitoring process.

As shown in Figure 3, twenty-nine monitoring points were designed and installed for the six pipelines; however, some monitoring points were abandoned because of the surface traffic requirement and other reasons, and only 15 of them were kept. Even worse, the pipeline settlement monitoring lagged behind the station tunnel construction and the ground surface settlement. Ground surface settlement already occurred when starting the monitoring of the pipeline settlement. Pipeline settlement monitoring ended in the case of holing-through of the tunnels because the monitoring points were covered to restore the road surface at that time.

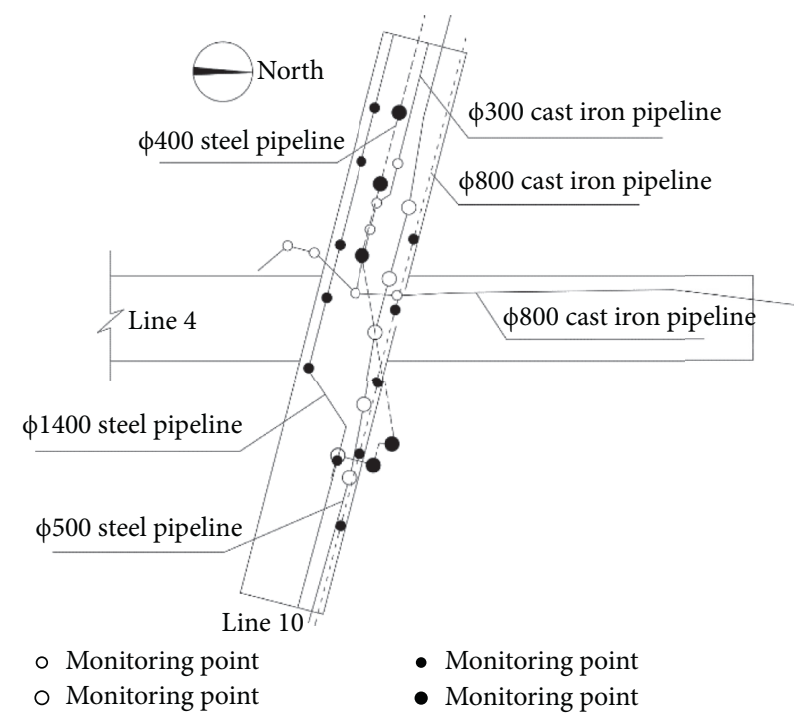

Figure 3: Plane view of the monitored pipelines.

3.2. Monitoring Results. Pipeline settlement monitoring lasted 322 days during the station tunnel construction, and settlement of the ground surface over the pipeline was also monitored in the meantime for comparison. After the tunnel construction was completed and the pipeline settlement became basically stable, the monitored results were regarded as the final settlements of the pipelines. The measured final settlements of the pipelines and the ground surface are shown in Table 3. A negative value in Table 3 denotes settlement.

Analysing the measured results, five construction cases were considered: case A: excavation face of the lower two drifts (drift 1 and drift 2) passing below the monitoring point; case B: excavation face of the upper two drifts (drift 3 and drift 4) passing below the monitoring point; case C: holing-through of the four drifts; case D: excavation face of the upper part of the tunnel passing below the monitoring point (corresponding to the step 4 presented in Figure 2(d)); and case E: holing-through of the tunnel and concreting of the bottom floor slab (corresponding to the step 6 presented in Figure 2(f)). Thus, the development of the tunnel construction induced pipeline settlements can be divided into four stages: (1) before case A (stage 1); (2) between case A and case $\mathrm{C}$ (stage 2); (3) between case $\mathrm{C}$ and case $\mathrm{D}$ (stage 3 ); and (4) between case D and case E (stage 4).

3.2.1. Measured Results of the $\phi 1400$ Steel Pipeline for the Water Supply. The plane view of the $\phi 1400$ steel pipeline orientation relative to the tunnel is presented in Figure 5. Six monitoring points were installed for this pipeline: WS14-1, WS14-2, WS14-3, WS14-4, WS14-5, and WS14-6. The monitoring point WS14-6 was abandoned during the construction, and the other five points were kept and used.

The measured results of the pipeline settlement at the five points and of the ground surface settlement are given in Figure 6. Figure 6(a) shows the development of the pipeline settlement over the construction time in terms of weeks; 
TABLE 2: Detailed information of the monitored pipelines.

\begin{tabular}{lcccccccc}
\hline No. & Type & Material & Diameter $(\mathrm{mm})$ & Cover depth $(\mathrm{m})$ & Wall thickness $(\mathrm{mm})$ & Joint & Section length $(\mathrm{m})$ & Installation time $(\mathrm{s})$ \\
\hline 1 & Water & Steel & 1400 & 2.26 & 12 & Weld & 6 & 1970 \\
2 & Water & Cast iron & 300 & 2 & 8 & Socket & 5 & 1980 \\
3 & Water & Steel & 800 & 2.55 & 5 & Weld & 6 & 1950 \\
4 & Gas & Steel & 400 & 1.41 & 5 & Weld & 6 & 9 \\
5 & Gas & Steel & 500 & 1.41 & 14.5 & Weld & 9 & 1980 \\
6 & Water & Cast iron & 800 & 1.5 & 18 & Socket & 5 & 1980 \\
\hline
\end{tabular}

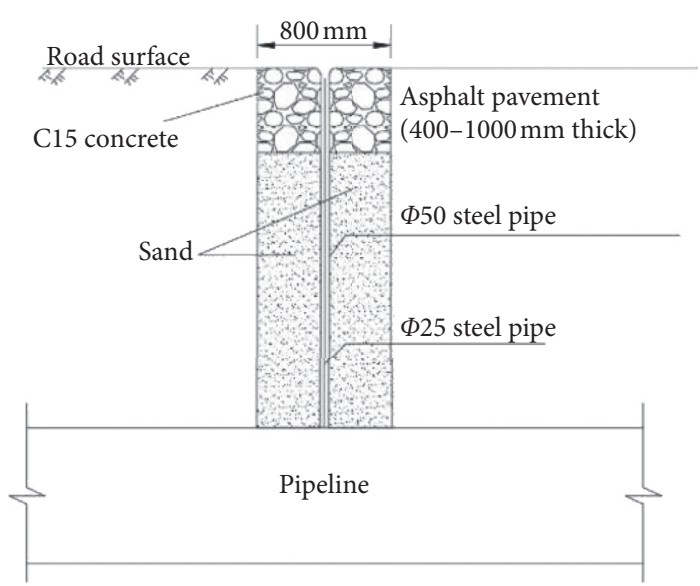

(a)

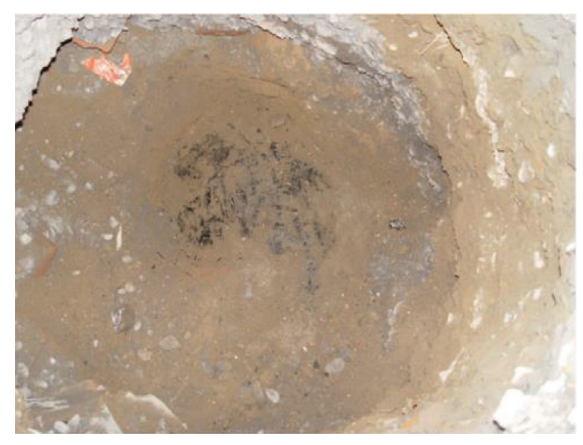

(b)

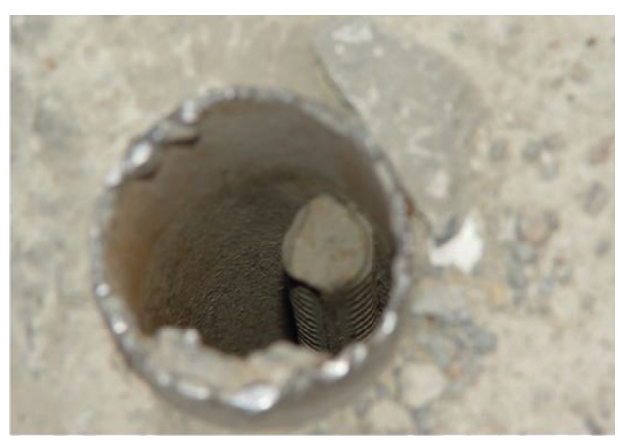

(c)

FIGURE 4: Pipeline settlement monitoring point: (a) installment of pipeline settlement monitoring point; (b) exposed pipeline surface; (c) the steel bar end at the road surface.

Figures 6(b)-6(f) provide both the pipeline settlement at the different monitoring points and the surface settlement over the pipeline. Cases A-E are also marked in Figures 6(b)-6(f) to clearly display the construction stages.

The Line 10 tunnel excavation was performed from both ends to the centre, and the pipeline settlement monitoring at the different monitoring points was delayed but began at almost the same time, resulting in varied settlement amounts that were not measured and the different final settlements at the different measuring points, as shown in Figure 6(a). The measured maximum settlement is $39.1 \mathrm{~mm}$ at the point WS14-5.

As shown in Figures 6(b)-6(f), the pipeline settlement monitoring lagged behind the surface monitoring and the tunnel construction because of the delay for the pipeline monitoring point installation, and some pipeline settlements were not measured. The varied increase rate in the different construction stages of the pipeline settlement is clearly displayed in Figures 6(b)-6(f) despite some pipeline settlement data being lost.

The pipeline settlement can be divided into four stages: (1) stage 1-initial slow increase when excavating the below two drifts; (2) stage 2-rapid increase when excavating the above two drifts until holing-through of the four drifts; (3) stage 3-remaining almost stable when excavating the upper part of the tunnel; and (4) stage 4-small increase when excavating the remaining part of the tunnel. This four-stage development trend is true of the ground surface settlement.

The initially monitored values of the pipeline settlement and the surface settlement approach zero when the excavation face is ahead and far from the monitoring point. As shown in Figures 6(e) and 6(f) and Table 3, when beginning to measure settlements at the points WS14-4 and WS14-5, the measured face settlements over the two points are 
TABLE 3: Measured final settlements of the pipelines and the ground surface.

\begin{tabular}{|c|c|c|c|c|c|}
\hline \multirow[b]{2}{*}{ Items } & \multicolumn{2}{|c|}{ Pipeline } & \multicolumn{3}{|c|}{ Ground surface over pipeline } \\
\hline & $\begin{array}{l}\text { No. of monitoring } \\
\text { point }\end{array}$ & $\begin{array}{l}\text { Settlement } \\
\quad(\mathrm{mm})\end{array}$ & $\begin{array}{l}\text { Final settlement } \\
(\mathrm{mm})\end{array}$ & $\begin{array}{l}\text { Settlement before measuring } \\
\text { pipeline settlement }(\mathrm{mm})\end{array}$ & $\begin{array}{c}\text { Concurrent } \\
\text { surface settlement } \\
(\mathrm{mm})\end{array}$ \\
\hline \multirow{5}{*}{$\begin{array}{l}\phi 1400 \text { steel water } \\
\text { pipeline }\end{array}$} & WS14-1 & -13.8 & -53.5 & -31.1 & -22.4 \\
\hline & WS14-2 & -34.2 & -65.0 & -29.5 & -35.5 \\
\hline & WS14-3 & -33.6 & -65.5 & -10.2 & -55.3 \\
\hline & WS14-4 & -31.4 & -59.0 & -5.9 & -53.1 \\
\hline & WS14-5 & -39.1 & -58.2 & -3.4 & -54.8 \\
\hline \multirow{3}{*}{$\begin{array}{l}\phi 300 \text { cast iron water } \\
\text { pipeline }\end{array}$} & WI3-1 & -37.6 & -71.3 & -31.7 & -39.6 \\
\hline & WI3-2 & -42.5 & -74.5 & -22.4 & -52.1 \\
\hline & WI3-3 & -44.6 & -56.8 & -6.0 & -50.8 \\
\hline $\begin{array}{l}\phi 800 \text { steel water } \\
\text { pipeline }\end{array}$ & WS8-3 & -35.1 & -59.8 & -3.6 & -56.2 \\
\hline \multirow{3}{*}{$\begin{array}{l}\phi 400 \text { steel gas } \\
\text { pipeline }\end{array}$} & GS4-1 & -32.7 & -59.4 & -29.0 & -30.4 \\
\hline & GS4-2 & -46.0 & -74.5 & -22.4 & -52.1 \\
\hline & GS4-3 & -32.9 & -61.1 & -9.6 & -51.5 \\
\hline $\begin{array}{l}\phi 500 \text { steel gas } \\
\text { pipeline }\end{array}$ & GS5-1 & -44.6 & -73.9 & -28.9 & -45 \\
\hline$\phi 800$ cast iron water & WI8-4 & -30.6 & - & - & - \\
\hline pipeline & WI8-3 & -47.5 & - & - & - \\
\hline
\end{tabular}

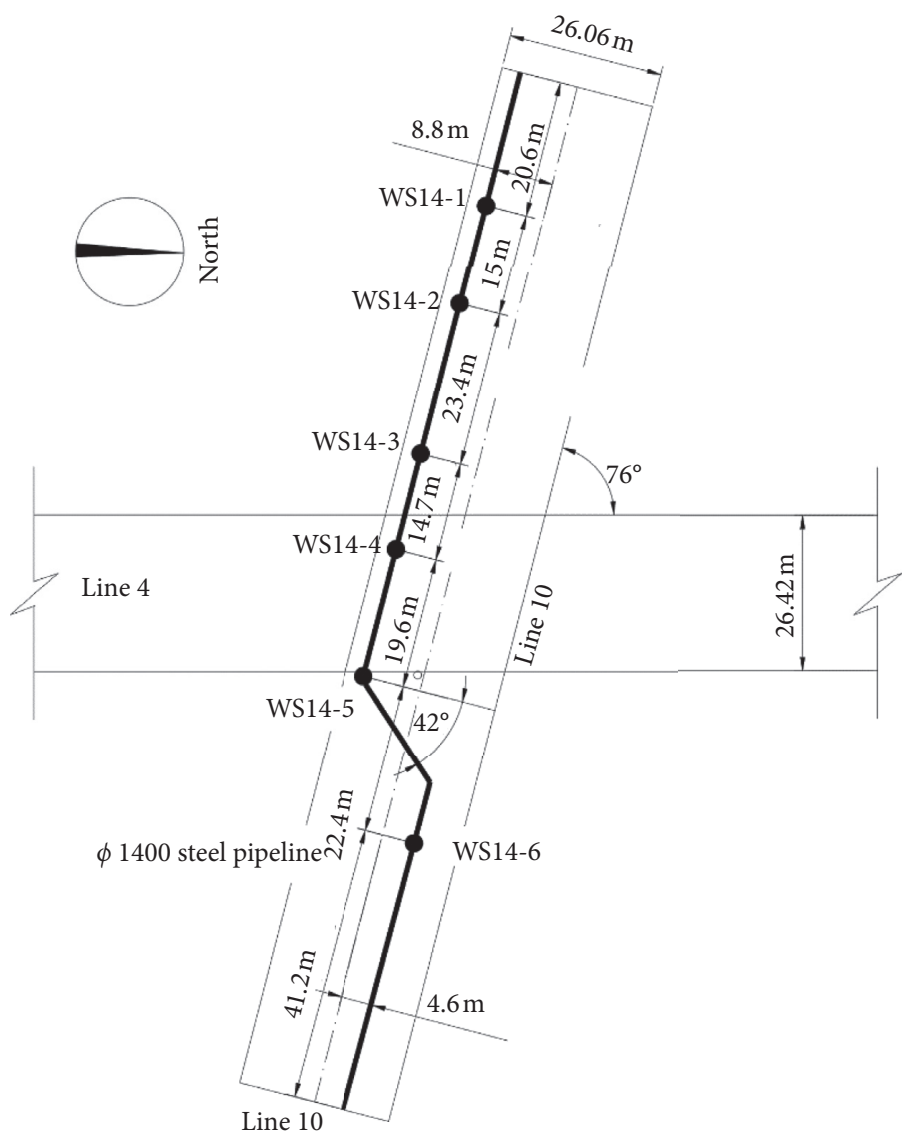

FIGURE 5: Monitoring point layout of the $\phi 1400$ steel water pipeline.

$-5.9 \mathrm{~mm}$ and $-3.4 \mathrm{~mm}$, respectively, which are smaller than the settlements of other points. Thus, the recorded results at the two points are closer to reality than those at other points. Taking the readings at WS14-4 and WS14-5 as examples, the variations in the pipeline and the surface settlements with the construction time are studied.

As shown in Figures 6(e) and 6(f), the pipeline settlement and the surface settlement increased at almost the same 


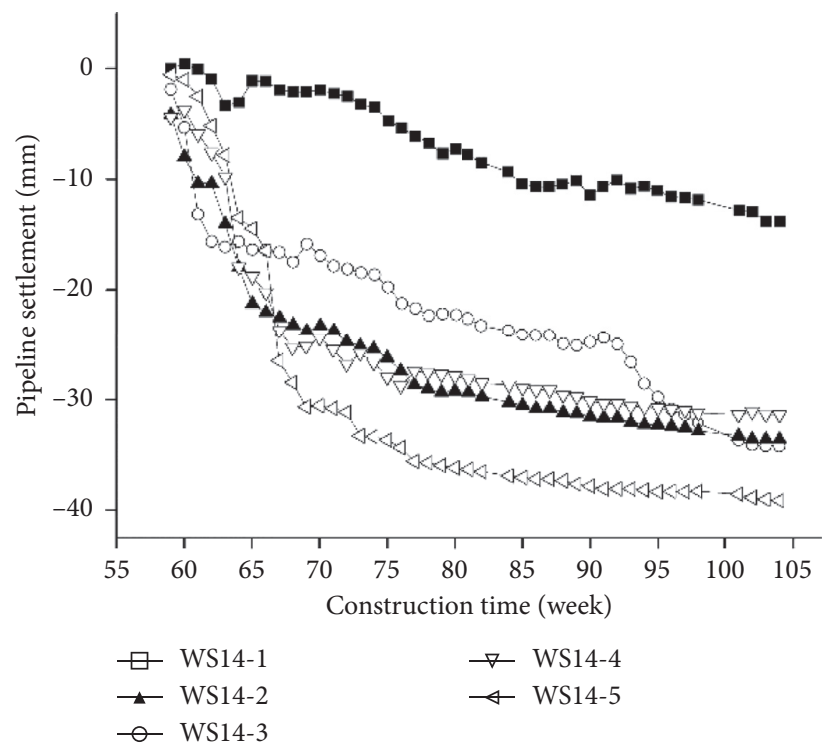

(a)

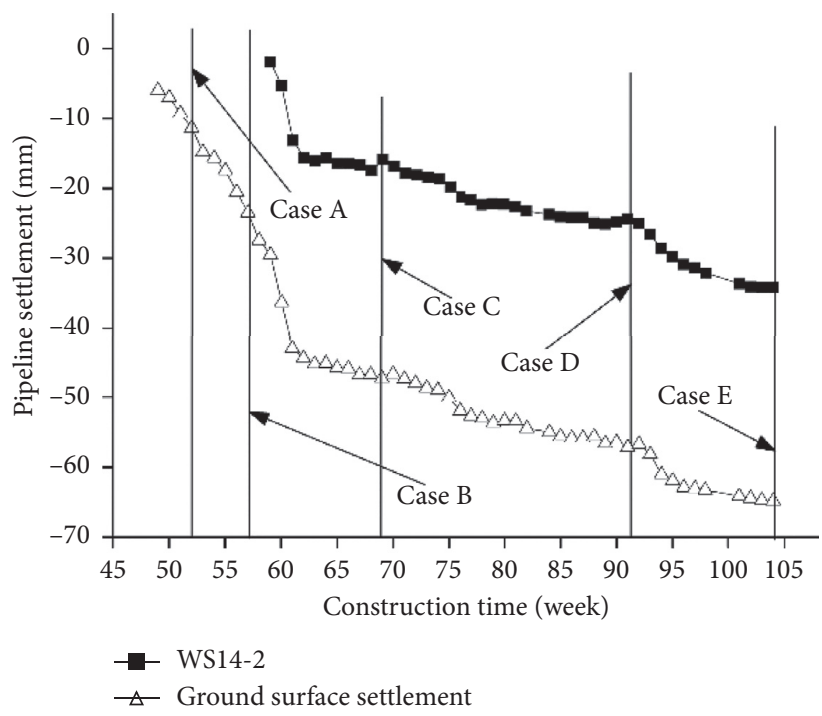

(c)

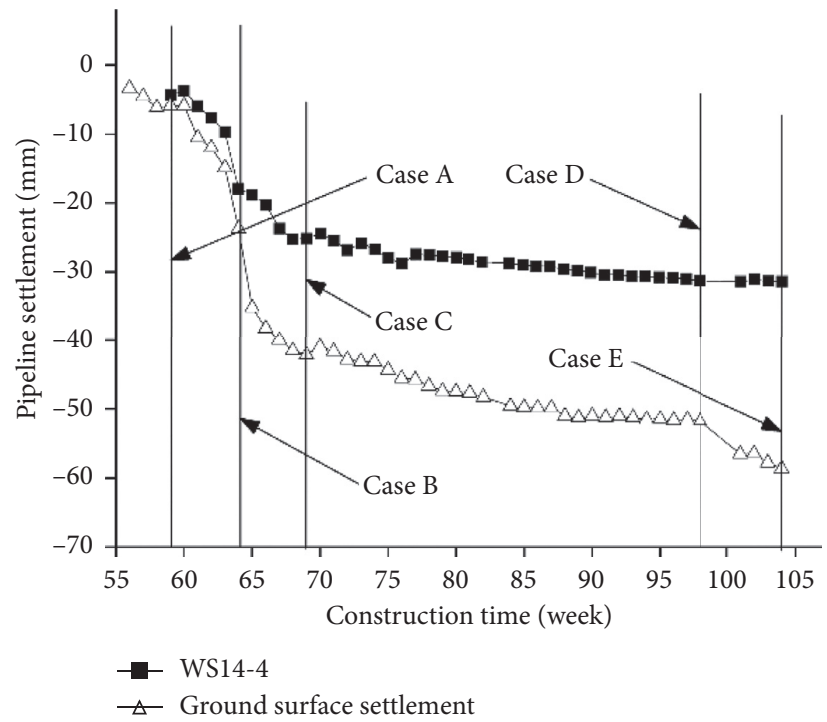

(e)

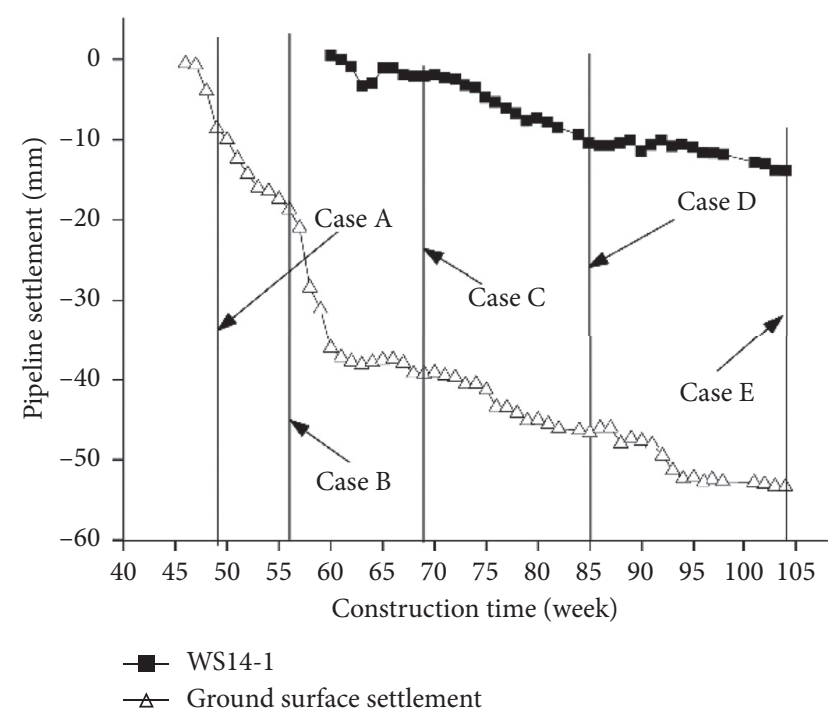

(b)

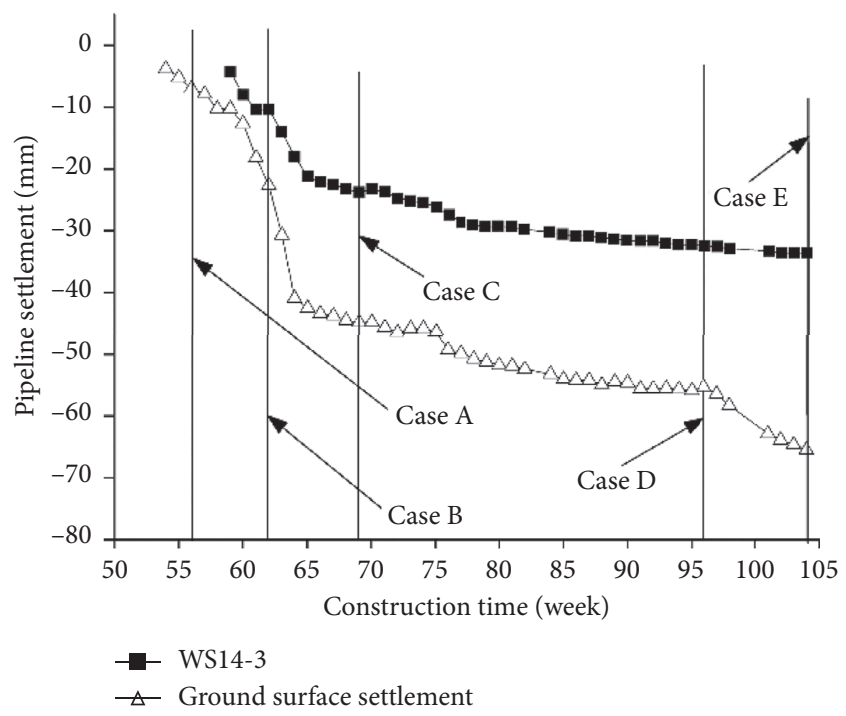

(d)

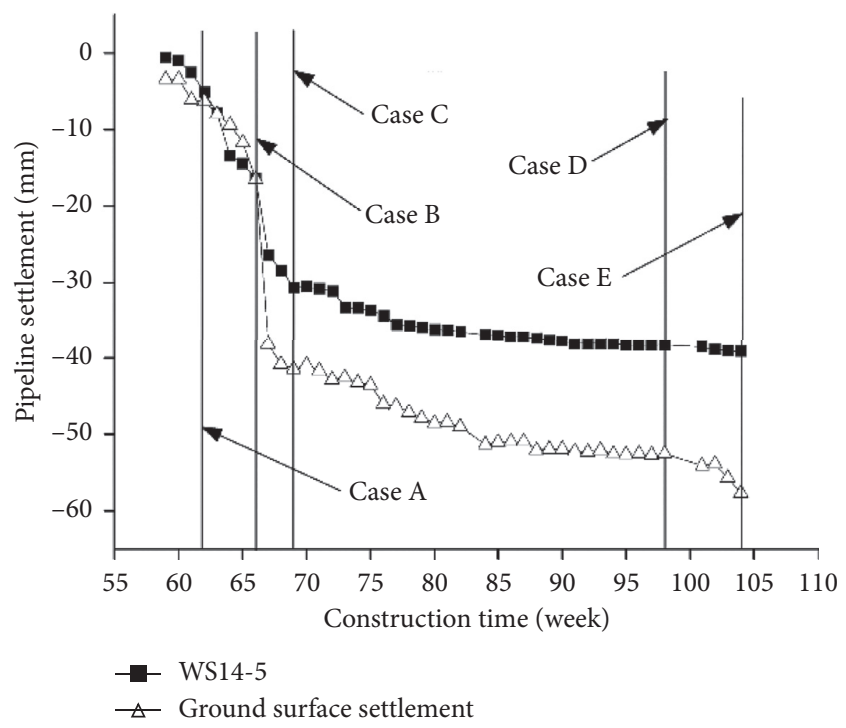

(f)

FIGURE 6: Variations in the settlements of the $\phi 1400$ steel water pipeline and the ground surface during the construction period: (a) measured pipeline settlement at five monitoring points; (b) settlements as WS14-1 and surface; (c) settlements as WS14-2 and surface; (d) settlements as WS14-3 and surface; (e) settlements as WS14-4 and surface; (f) settlements as WS14-5 and surface. 
small rate at the beginning of the tunnel construction and continued to increase until the beginning of excavation of the above two drifts. With the sustained excavation of the above two drifts, a large difference resulted between the ground face settlement and the pipeline settlement. When holing-through the four drifts (drifts 1-4), the differences reached $20.6 \mathrm{~mm}$ and $14.3 \mathrm{~mm}$ at WS14-4 and WS14-5, respectively. This trend intensified with the excavation of the remaining part, resulting in the final differences of $27.3 \mathrm{~mm}$ and $18.6 \mathrm{~mm}$ for WS14-4 and WS14-5, respectively. If a pipeline follows the green field movement, its settlement or concurrent settlement must be larger than the ground surface settlement; however, vice versa is found in the measured results, as listed in Table 3, leading to the conclusion that separation between the pipeline and the surrounding soil must exist.

3.2.2. Measured Results of the $\phi 800$ Steel Pipeline for the Water Supply. As shown in Figure 7, five monitoring points were arranged along the $\phi 800$ steel water pipeline, but only the point of WS8-3 was kept and used during the construction. The variations in settlements of the $\phi 800$ steel water pipeline and the above ground surface with the construction time in terms of weeks are presented in Figure 8.

When initially monitoring the pipeline settlement, the above ground surface settlement was approximately $3.6 \mathrm{~mm}$, and the occurred pipeline settlement was negligible at that time.

The measured data at WS8-3, shown in Figure 8, display the variations in the pipeline settlements with the construction time; these variations follow a law of four-stage development. Pipeline settlement achieved the rapid increase in stages 2 and 4, whereas the increase in pipeline settlement was small or even approached zero during stages 1 and 3 .

The rapid increases in the pipeline settlement and the surrounding soil settlement cause a swift separation of the pipeline from its soil beneath, resulting in a difference between the pipeline settlement and the above ground surface settlement. The difference of approximately $16 \mathrm{~mm}$ for $\Delta_{1}$ in Figure 8 remained almost unchanged during excavating the remaining part of the side drift and reached approximately $24 \mathrm{~mm}$ when finishing the excavation of the tunnel. The actual separation, $\Delta_{2}$ in Figure 8, should be larger than $\Delta_{1}$. In Figure 8 , the dashed line represents the inferred settlement of the soil beneath the pipeline.

\subsubsection{Measured Results of the $\phi 300$ Cast Iron Pipeline for the} Water Supply. As presented in Figure 9, three monitoring points denoted as WI3-1, WI3-2, and WI3-3 were installed on the $\phi 300$ cast iron pipeline for the water supply. All these three points were kept and used in the construction.

The measured data of the $\phi 300$ cast iron pipeline are listed in Figure 10. This pipeline is approximately parallel to and over the centreline of the Line 10 tunnel. Although delayed monitoring exists at the three points of WI3-1, WI3-
2 , and WI3-3, four stages of the pipeline settlement development can be found. The recorded data at the three points displayed more or less the same development law if the delayed monitoring is considered. The separation of the pipeline from its soil beneath can also be concluded, and the difference between the pipeline settlement and the above ground surface settlement is approximately $22 \mathrm{~mm}$. As shown in Figure 10(d), small increase in settlement at WI 3-3 was recorded with the excavation of the lower section, possibly because of the improved ground.

\subsubsection{Measured Results of the $\phi 800$ Cast Iron Pipeline for the} Water Supply. As given in Figure 11, four monitoring points were arranged along the $\phi 800$ cast iron pipeline for the water supply; however, only two of them played their roles: WI8-3 and WI8-4. Because of the above ground face monitoring point far away from the two pipeline monitoring points, only the recorded results of the pipeline settlement are given in Figure 12.

The point of WI8-4 in the north of the Line 10 tunnel was mainly under the influence of excavation of the four drifts; the point of WI8-3 in the middle of the Line 10 tunnel was mostly affected by the construction of the middle drift.

Before excavating the above two drifts, the measured results at WI8-3 were larger than those at WI8-4. With the sustained advance of the above two drifts, the settlements at WI8-4 increased at a higher rate. After holing-through the four drifts, the settlements at WI8-4 exceeded those at WI83. The trend continued; however, the settlements at WI8-4 experienced a rapid increase with the excavation of the middle drift.

Similar to the above situation, the pipeline settlements at the two points displayed more or less the same trend of the four-stage development.

3.2.5. Measured Results of the $\phi 400$ Steel Pipeline for Gas Transportation. The designed settlement monitoring points of the $\phi 400$ steel pipeline for gas transportation are shown in Figure 13. Six points were arranged initially, but only three of them were employed to monitor the pipeline settlement. The three points are GS4-1, GS4-2, and GS4-3.

The $\phi 400$ steel gas pipeline lying approximately in the middle of the Line 10 tunnel was mostly affected by excavating the middle drift. The measured settlements at the three monitoring points are listed in Figure 14 together with the recorded above ground surface settlements. Similar pipeline settlement variations with the construction time can be found. The four-stage development law is also true of the $\phi 400$ steel gas pipeline.

After holing-through the four drifts, the difference between the surface settlement and the pipeline settlement at GS4-1 and GS4-2 was approximately $6 \mathrm{~mm}$ and reached $28 \mathrm{~mm}$ with the completion of the tunnel excavation. The recorded settlement at GS4-3 changed little over the period of excavating the middle drift; this behaviour is perhaps related with the local geology. 


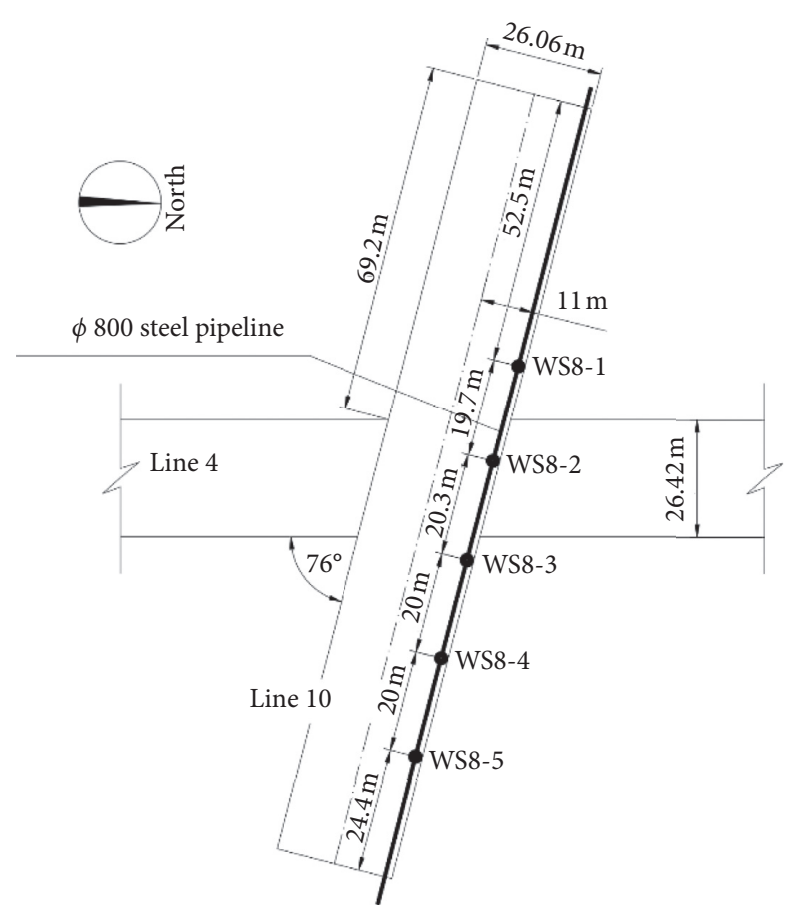

FIGURE 7: Monitoring point layout of the $\phi 800$ steel water pipeline.

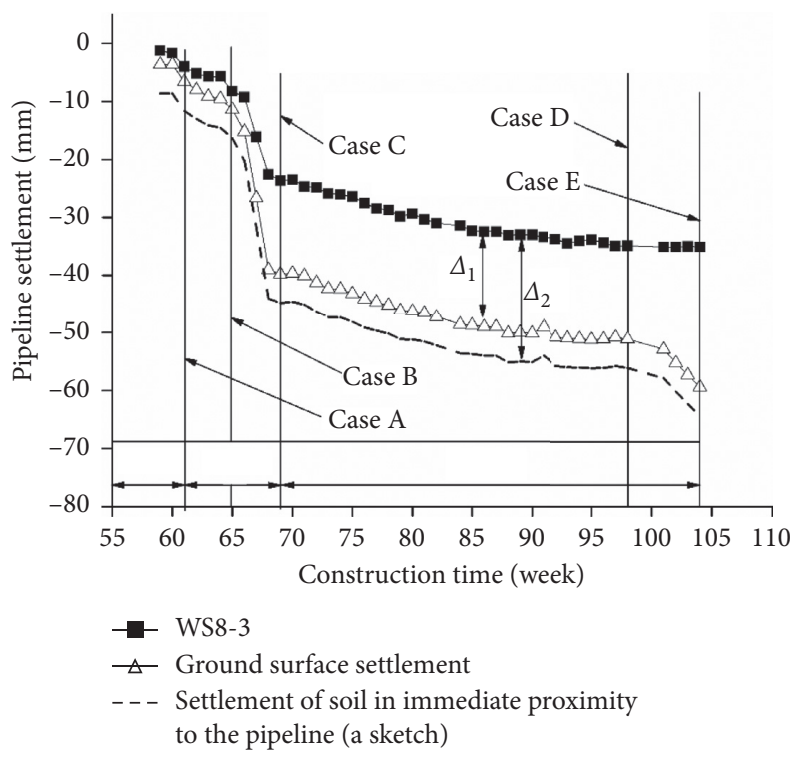

Figure 8: Pipeline settlement, ground surface settlement, and inferred settlement of the soil beneath the pipeline.

3.2.6. Measured Results of the $\phi 500$ Steel Pipeline for Gas Transportation. As shown in Figure 15, five monitoring points were installed for the $\phi 500$ steel pipeline for gas transportation; however, only one point of GS5-1 was kept and used in the construction.

The $\phi 500$ steel gas pipeline, located in the north of the Line 10 tunnel, was mostly affected by excavating the four drifts. Variations in the pipeline settlement at GS5-1 and the above surface settlement with the construction time are displayed in Figure 16. Similar to the above discussion, the pipeline settlement underwent three stages of

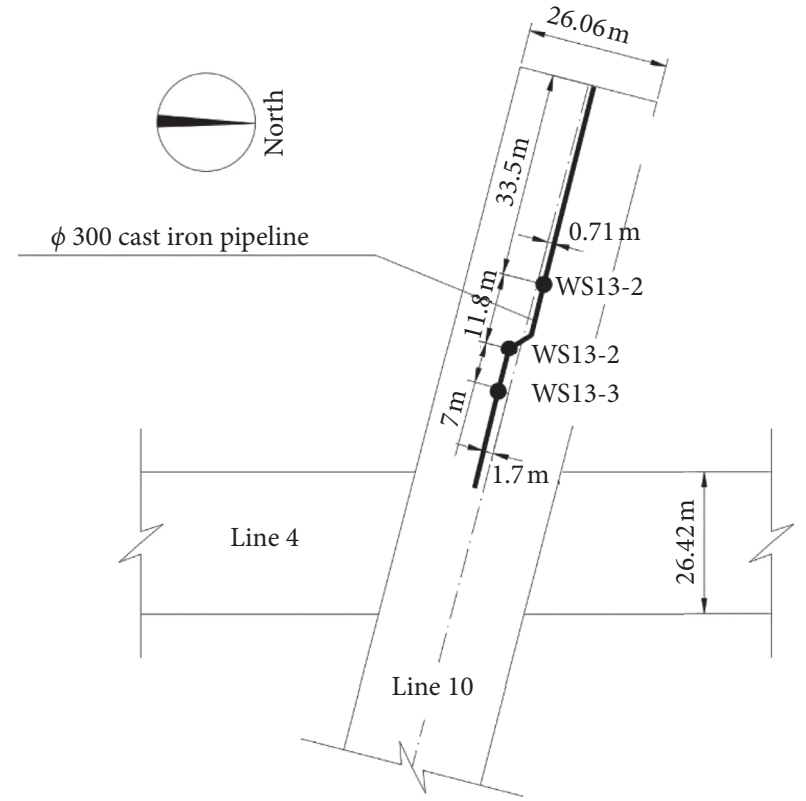

FIGURE 9: Monitoring point layout of the $\phi 300$ cast iron water pipeline.

development, and separation of the pipeline from its soil beneath occurred.

\section{Discussion}

4.1. Pipeline Settlement Development with the Tunnel Construction. The buried pipelines were affected by the soil settlement caused by the station tunnel construction. The pipeline settlement developed with the consecutive construction steps. The increase in pipeline settlement in different construction steps varied. The pipeline monitoring, though delayed, clearly displayed the varied increase over the different construction periods.

Referring to Figures 6, 8, 10,12,14, and 16, the pipeline settlement development occurred in four stages: (1) stage 1 , stage 2 , stage 3 , and stage 4 . Stage 1 refers to the period of excavating the below two drifts (drift 1 and drift 2); in this stage, the settlement increase is not fast. Stage 2 denotes the period from the excavation of the above two drifts (drift 3 and drift 4) to holing-through of the four drifts; in this stage, a sharp increase in pipeline settlement occurs. Stage 3 is defined as the period of excavating the upper part of the station tunnel under the protection of the top arched support; in this stage, the pipeline settlement increase is slow. Stage 4 is the time spent on constructing the remaining part; in this stage, some increase in pipeline settlement occurs at the beginning and then tends to be stable. This four-stage development law is also true of the ground surface settlement.

4.2. Pipe-Soil Separation. Determining how to tackle the pipe-soil interaction remains a critical task when evaluating the effects of the tunnelling-induced ground movements on the buried pipelines. The pipe-soil interaction is complex 


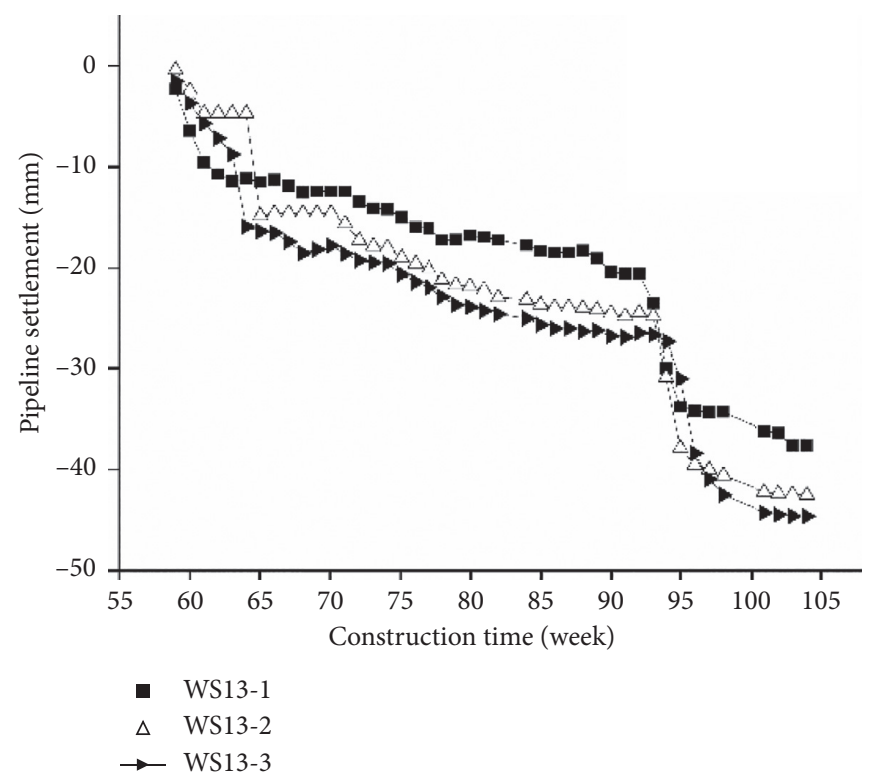

(a)

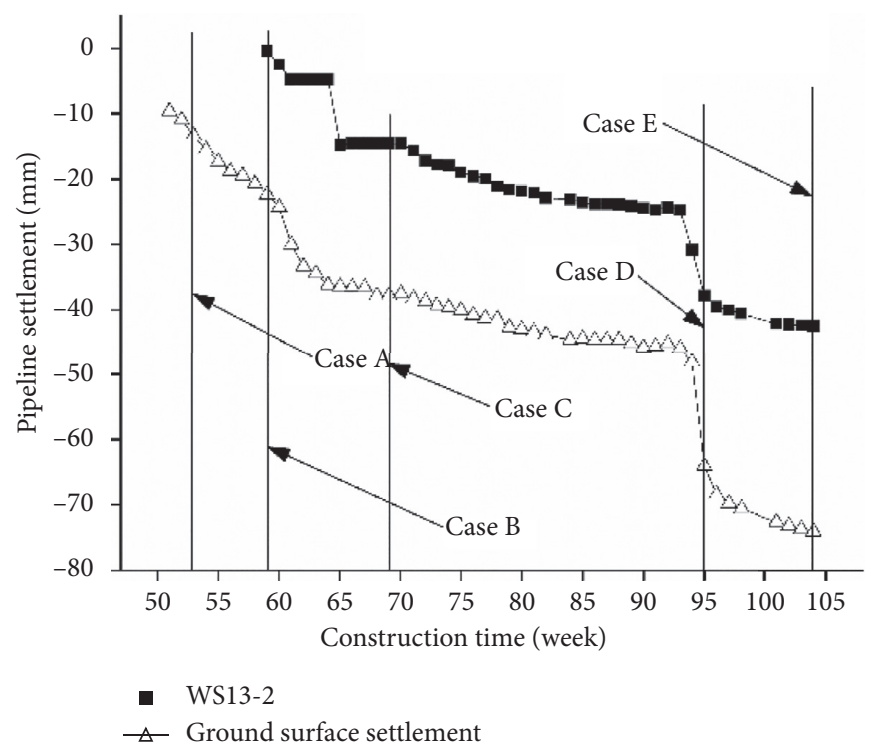

(c)

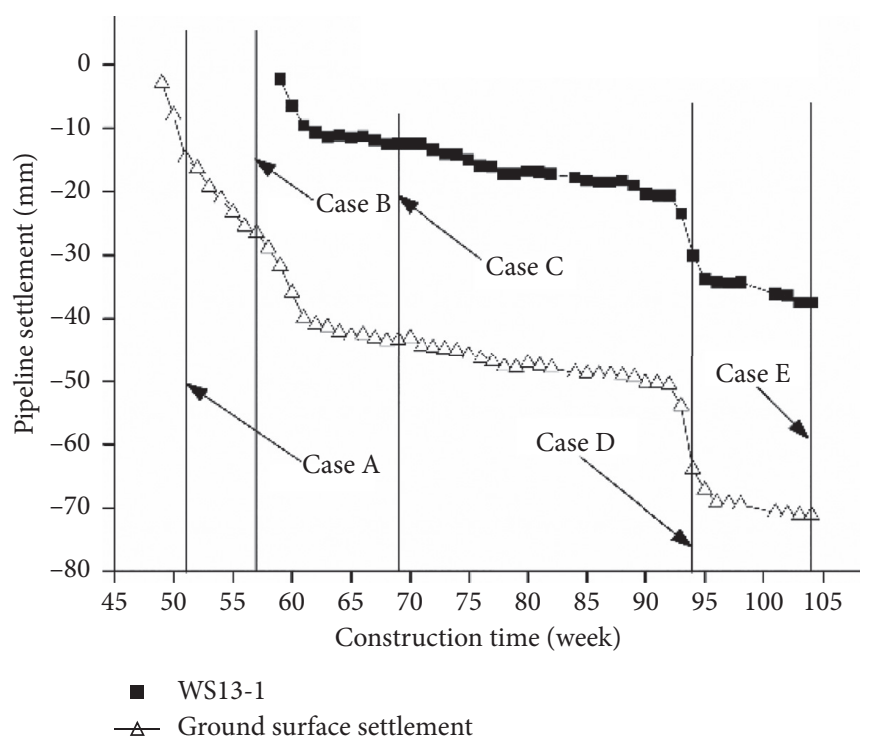

(b)

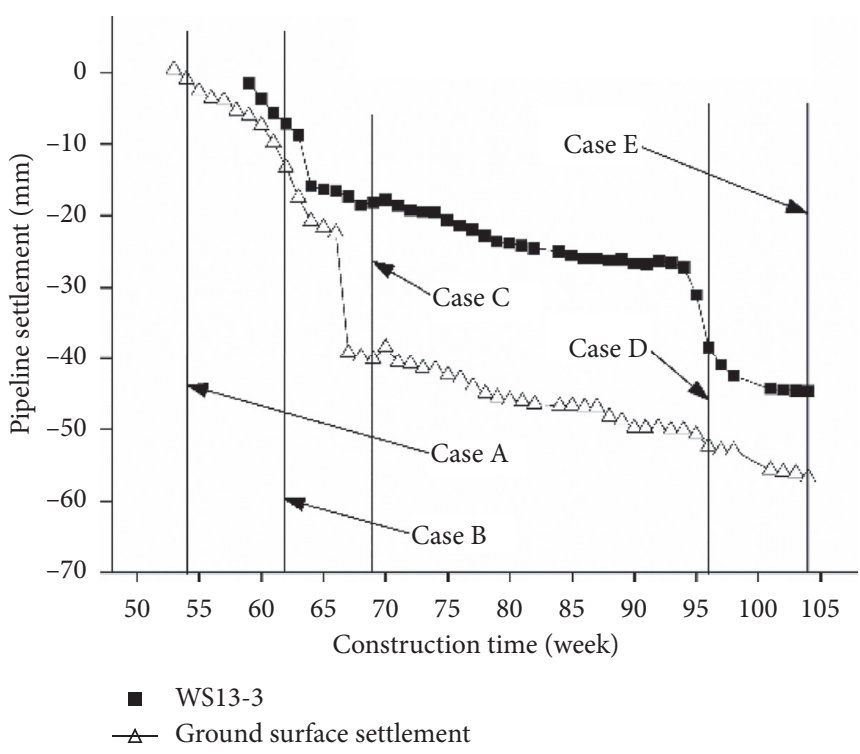

(d)

FIGURE 10: Variations in the settlements of the $\phi 300$ cast iron pipeline and the ground surface with the construction time: (a) measured pipeline settlement at five monitoring points; (b) settlements at W13-1 and surface; (c) settlements at W13-2 and surface; (d) settlements at W13-3 and surface.

and influenced by many factors, such as the pipe diameter, the pipe material, the pipe burial depth, and the relative pipe-soil stiffness. The easy-to-use assumption that the pipeline is always in contact with the soil is usually adopted in analytical methods, such as Winkler-based methods (e.g., Attewell et al. [3], Wang et al. [5], and Marshall et al. [13]) and continuum methods (e.g., Klar et al. [8-10] and Vorster et al. [11]). If a pipeline is always in contact with the soil and follows the soil displacement, then its settlement must exceed the overground surface settlement for the settlement propagates upward in the case of a tunnel excavation below the pipeline. However, the in situ measured settlements of the pipelines and the ground surface do not satisfy the assumption. Although the pipeline settlement being always less than the ground surface settlement measured at the same time may be attributed to the delayed pipeline settlement monitoring, the concurrent pipeline settlement is always less than the ground surface settlement, as shown in Table 3. This finding can account rationally for the separation of the pipeline from the soil beneath.

From the details of the pipelines listed in Table 2, the pipes (steel or cast iron in material and $300-1400 \mathrm{~mm}$ in diameter) are more or less rigid. The almost rigid pipelines are liable to resist the above distributed loads imposed through the settled soil when tunnelling-induced 


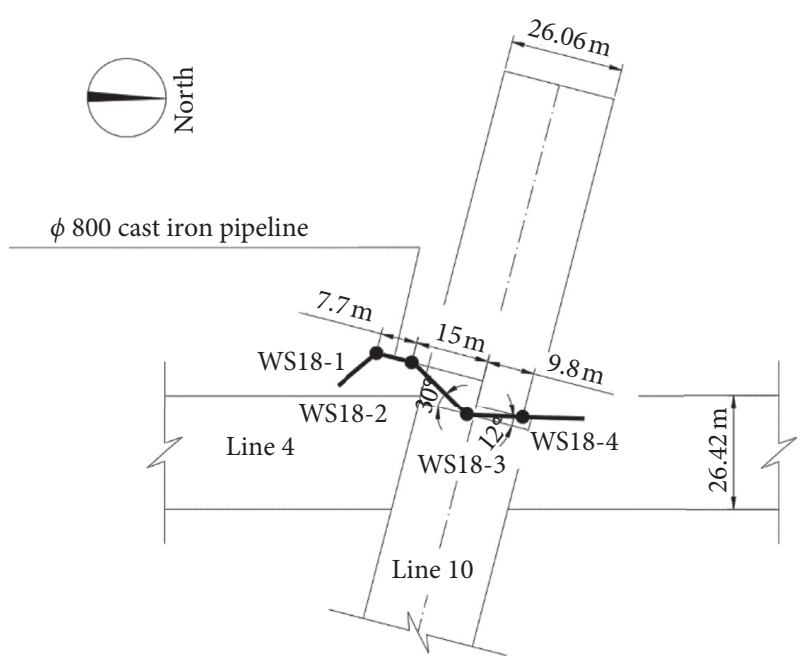

FIGURE 11: Monitoring point layout of the $\phi 800$ cast iron water pipeline.

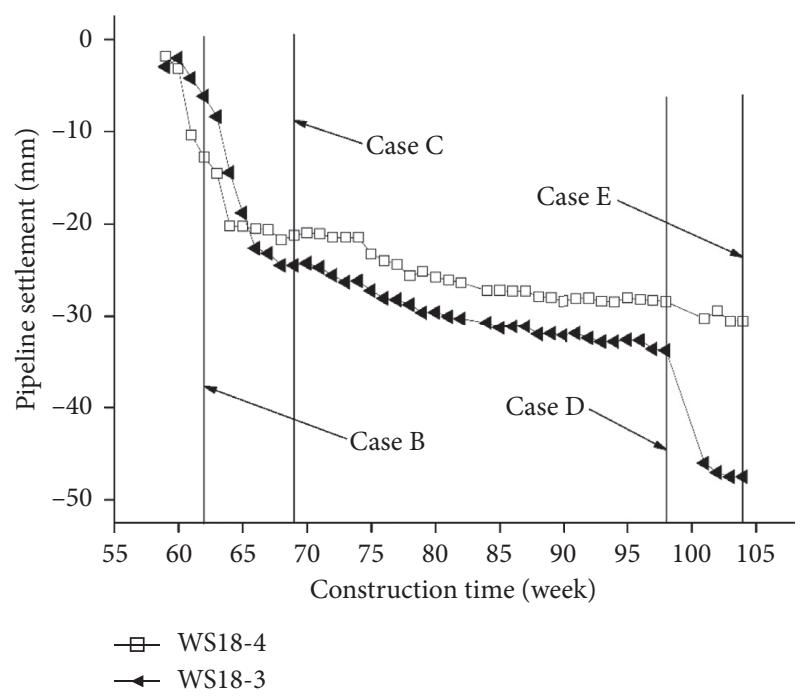

FIGURE 12: Variation in settlements of the $\phi 800$ water cast iron pipe with construction time.

settlements occur in the soil surrounding the existing pipelines, causing separation of the pipelines from the soil beneath.

Because of the difficulty in measuring separation between the pipeline and its beneath soil, the difference between the concurrent ground surface settlement and the recorded pipeline settlement, listed in Table 3, can be regarded as an estimator of the separation amount. The larger the difference, the larger the separation amount. Note that the positive difference at GS4-1 was discarded; this difference is perhaps is related with the local soil and the pipeline conditions.

The variations in the calculated settlement difference with the pipe diameter and the pipe burial depth are plotted in Figure 17. The data points in Figure 17 reveal that, generally speaking, the larger the pipe diameter and the pipe burial depth, the larger the resistance to the overlying soil of

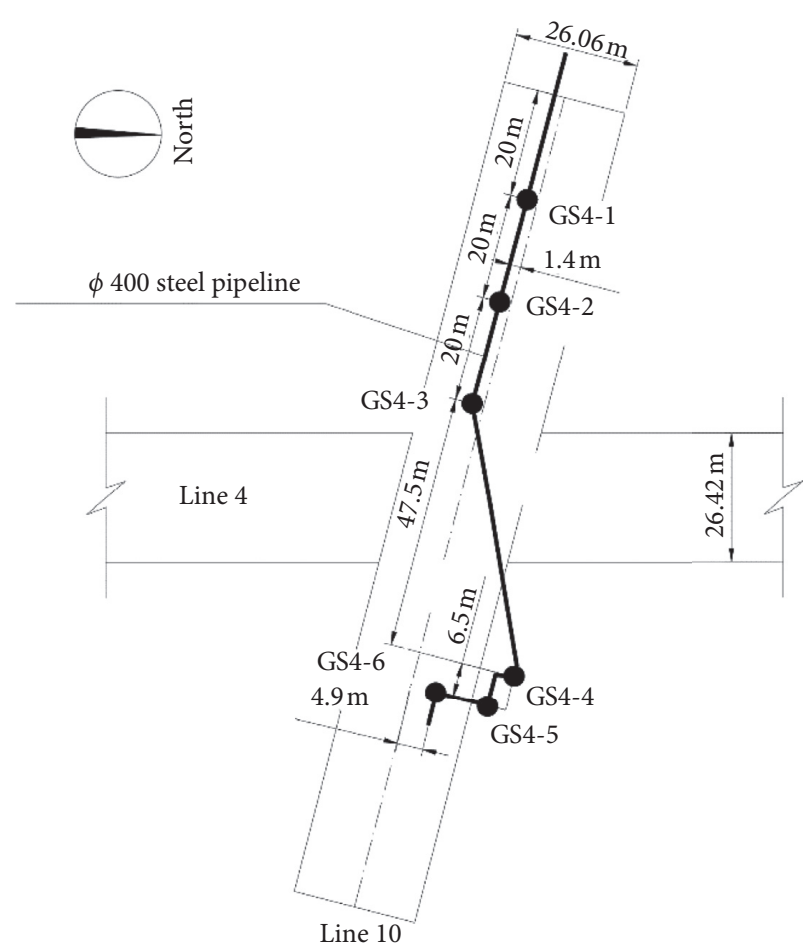

FIGURE 13: Monitoring point layout of the $\phi 400$ steel gas pipeline.

the pipeline. Besides the pipe diameter and burial depth, the calculated settlement of the pipeline varies with many other factors, such as soil type and moisture, pipe thickness, and pipe joint stiffness. But generally speaking, despite some anomalies, the calculated settlement decreases with the increasing the pipe diameter and burial depth, as plotted in Figure 17. When a pipe is buried at a shallow depth, a gap can be formed easily when it is subjected to differential ground motion. This is called the bridging effect. At a greater depth, the pipe deformation will more conform to the soil deformation. Thus, for steel or cast iron pipes with diameters more than $400 \mathrm{~mm}$ and burial depth more than $1.8 \mathrm{~m}$, the separation of the pipeline from its soil beneath almost surely exists. When modelling tunnelling influences on these types of pipelines, the use of a deformation compatibility at the pipe-soil interface should be well evaluated; otherwise, overestimation of the pipeline settlement and bending moment will be made. Therefore, rather than using the theoretical method directly, it would be a better assessment of the response of the buried pipelines to consider the soilpipe interaction in more realistic conditions of soil-pipe separation.

4.3. Indirect Pipeline Monitoring. Monitoring buried pipelines is a demanding and painstaking task because excavating the road surface to expose pipeline surface is not allowed in uttermost cases in urban areas according to the demand for maintaining surface traffic. Of course, the excavation work itself in the particular circumstances limits the use of the direct monitoring of pipeline settlement. For large-diameter and deeply buried pipelines, the above 


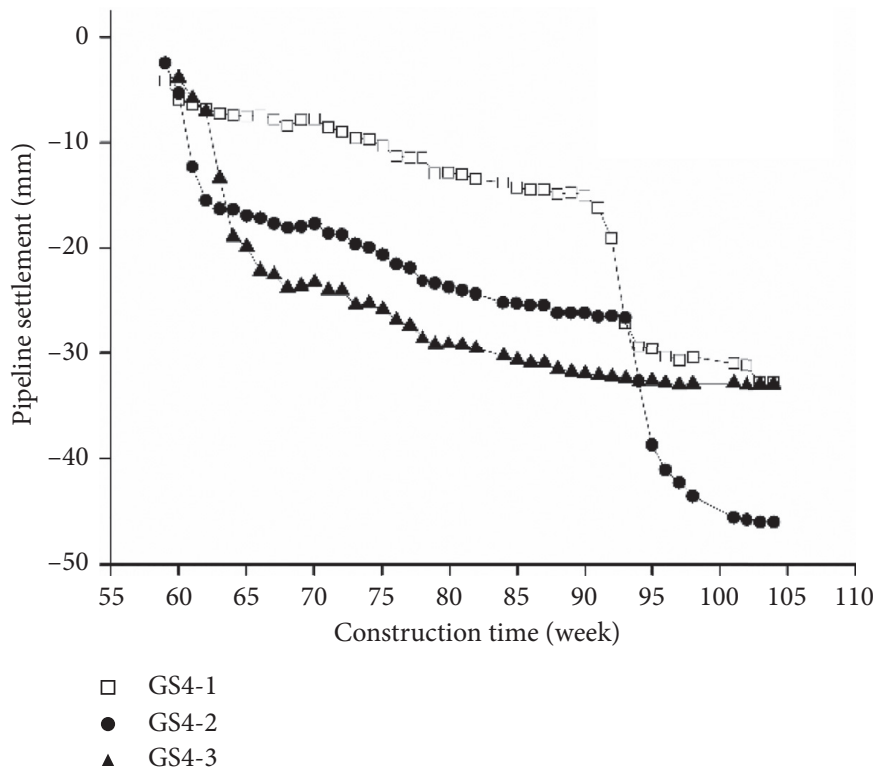

(a)

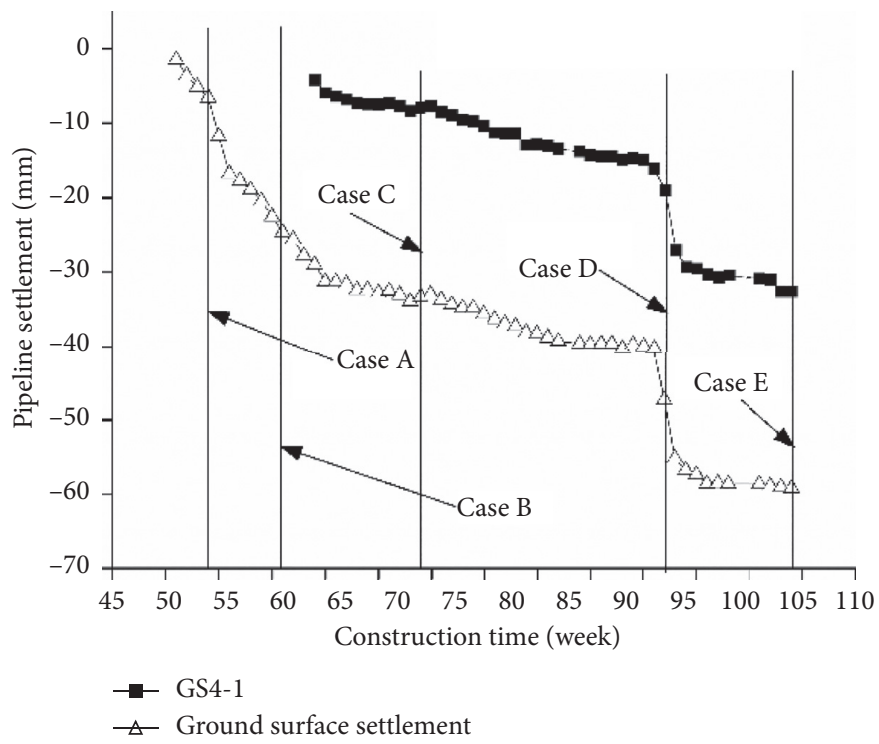

(b)

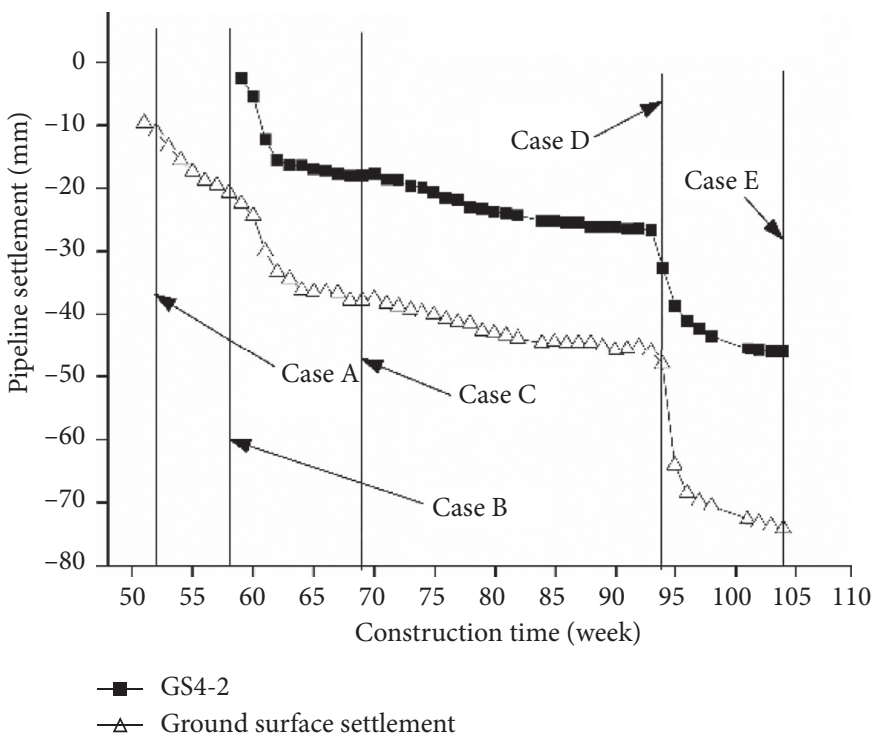

(c)

Figure 14: Continued. 


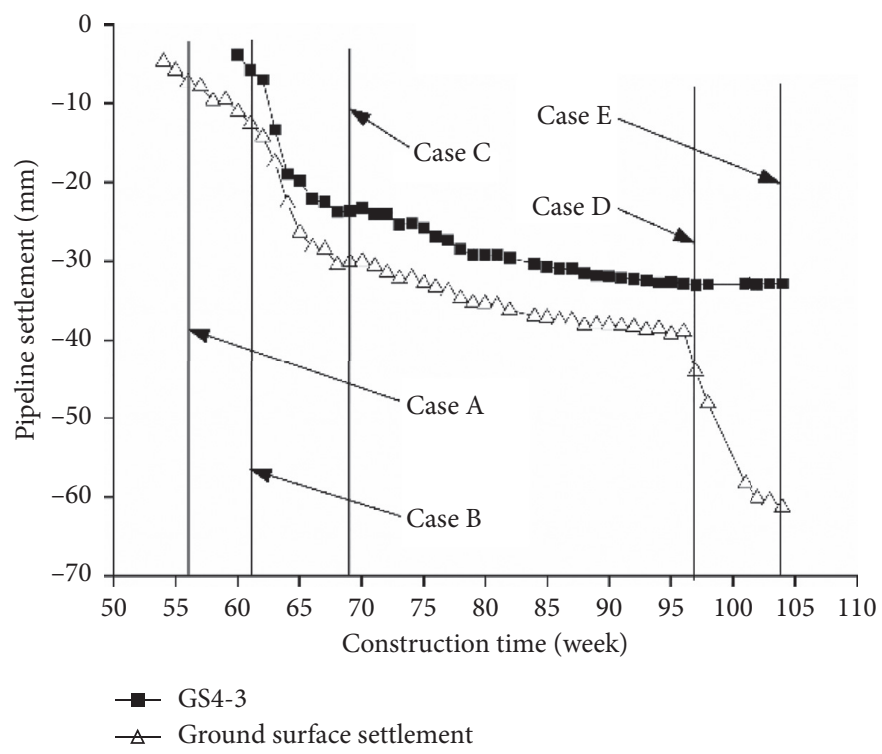

(d)

FIgURE 14: Variations in the settlements of the pipeline and the above ground surface with the construction time: (a) measured pipeline settlement at three monitoring points; (b) settlements at GS4-1 and surface; (c) settlements at GS4-2 and surface; (d) settlements at GS4-3 and surface.

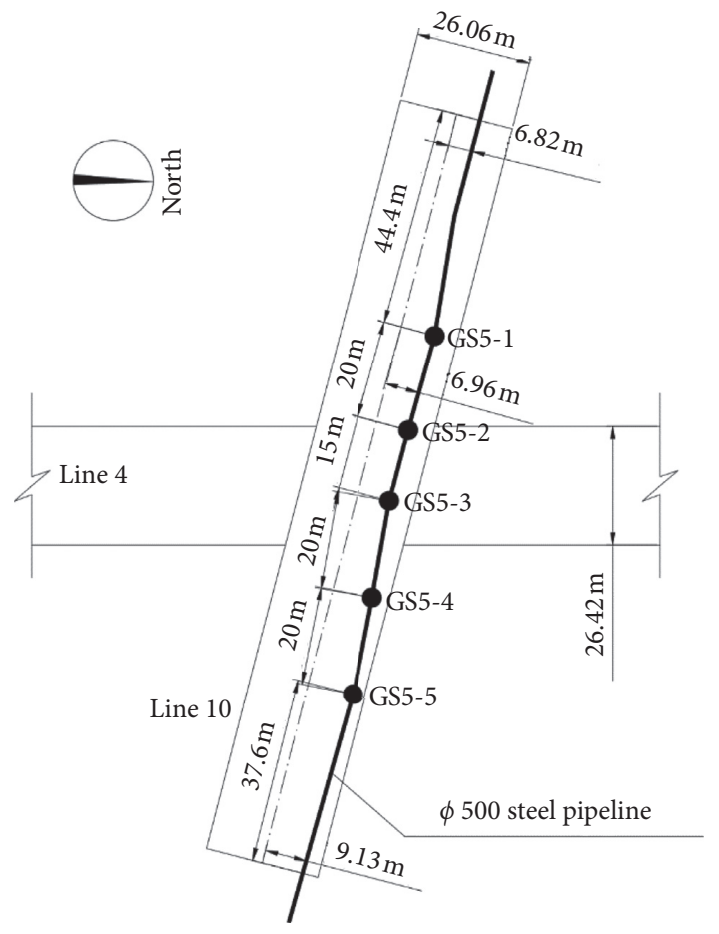

FIGURE 15: Monitoring point layout of the $\phi 500$ steel gas pipeline.

finding that the pipeline will be separated from the soil beneath has a practical implication because the surface settlement will exceed the pipeline settlement and can be taken as an estimator of the pipeline settlement. In that case, the ground surface settlements are monitored instead of the pipeline settlements. Because the monitored results are larger than the pipeline settlements, the pipeline safety can be conservatively evaluated. This indirect pipeline monitoring truly saves time and decreases the cost; most important of all, this monitoring has negligible disruption of the surface traffic, representing a highly efficient and lowcost solution.

4.4. Allowable Deformations of Buried Pipelines. The safety of the buried pipelines as affected by adjacent tunnelling activities has been a main concern in ground space utilization, and establishing the pipeline deformation criteria to restrict the induced deformations of the existing pipelines is of paramount importance to guarantee the pipeline safety in construction. However, an essential feature, as far as allowable deformations of buried pipelines are concerned, is that the risk of a pipeline failure must be set in the context of overall pipeline reliability from all causes. Allowable deformations of the buried pipelines depend on a variety of issues, such as pipe material, joint configuration, pipeline age, pipeline function (e.g., gas or water), and loading history. In addition to the circumferential limits, initial conditions of the buried pipelines should also be taken into account to decide the allowable deformations. The tolerances of buried pipelines to tunnelling-induced ground movements are influenced by so many factors, few of which the tunnel designer has knowledge of because of the pipeline long history and other social reasons. No comprehensive criteria exist for relating construction-induced soil displacements with the safety and service of utilities. Under these circumstances, attempting to establish empirical criteria is reasonable, irrespective of the complex tunnel-soilpipe interaction and the many perplexing influencing factors. 


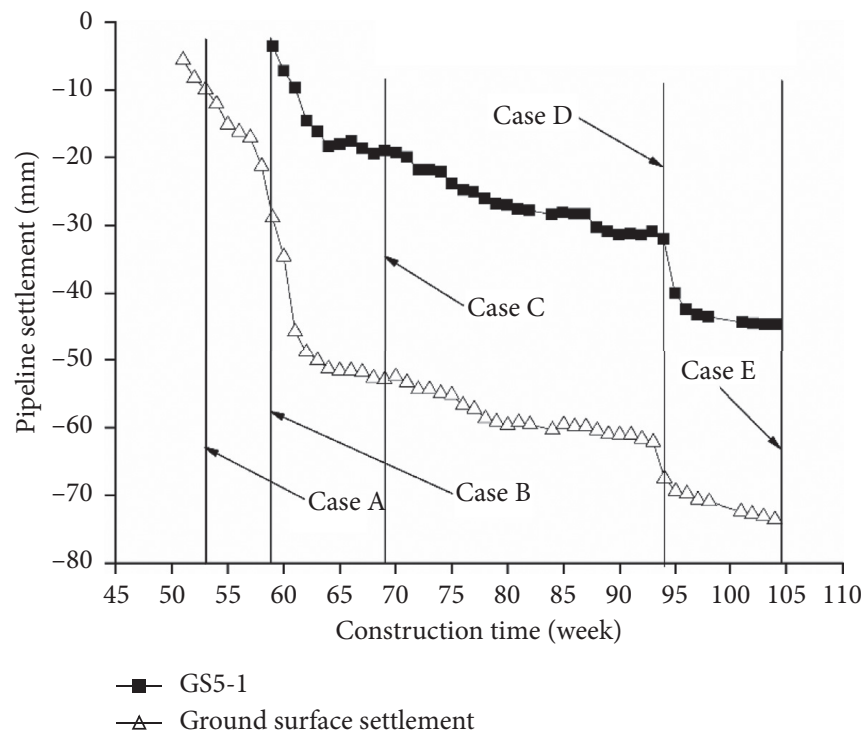

Figure 16: Variations in settlements of the pipeline and the above ground surface with the construction time.

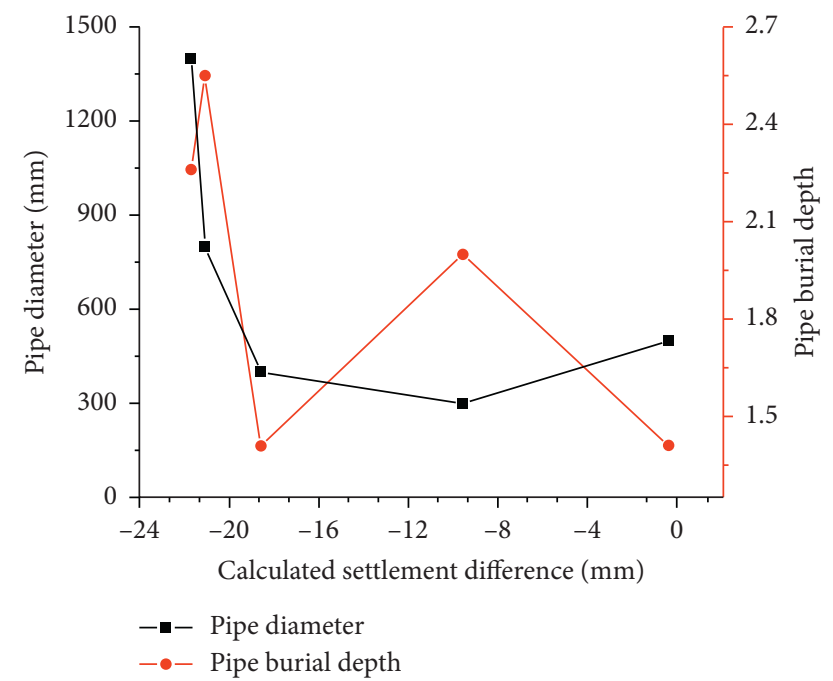

Figure 17: Calculated difference versus the pipe diameter and the pipe burial depth.

The attempted criteria, as presented by Bracegirdle et al. [4], can be divided three types: (1) empirical ground deformation criteria; (2) joint rotation and pull out criteria; (3) pipe strain criteria. From a monitoring point of view, the latter two types of criteria are impractical in practice and are not discussed in the paper.

For buried cast iron pipelines, O'Rourke and Trautman [34] presented the ratio of the maximum surface settlement $\delta_{\mathrm{m}}$ to the trough width parameter $i$ associated with excessive distortion as a function of pipe diameter, as shown in Figure 18, based on the assumption that mains composed of relatively large-diameter pipes would behave as rigid systems and mains composed of relatively small-diameter pipes would behave as either flexible or semiflexible systems, depending on the moment restraint characteristics of the joints. This proposed plot does provide a useful criterion for

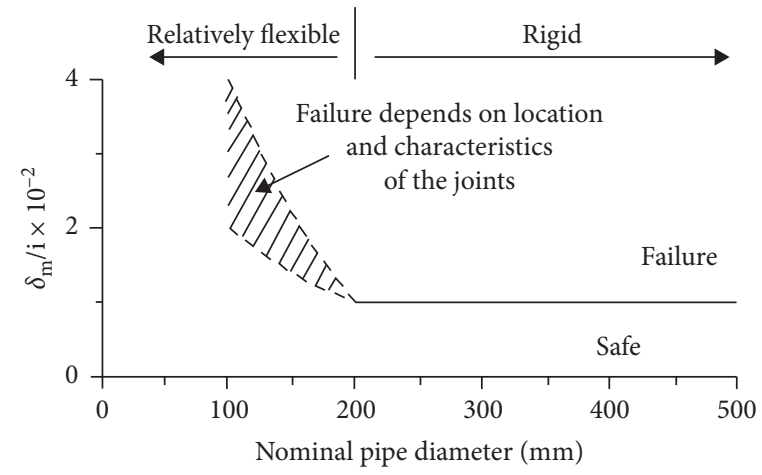

FIGURE 18: Relationship between the dimensionless settlements associated with pipeline damage and pipe diameters (from O’Rourke and Trautman [34]).

preliminarily and empirically determining the likelihood of damage to service pipelines. However, such important factors as pipe condition and type are not taken into consideration. In particular, no allowance was made for very sensitive services, such as large-diameter gas pipelines in cast iron or steel, where the consequences of pipeline damage may be very severe.

In practice, ground surface settlement is often used to evaluate tunnelling-induced effects on surrounding environment and can be employed to evaluate pipeline safety in the close vicinity. A preliminary assessment of the possible effect of tunnelling-induced movement can be conducted based on maximum surface settlements listed in Table 4 and suggested by Attewell et al. [3]. Nevertheless, the listed data were drawn from the majority of the tunnel construction of less than $2.5 \mathrm{~m}$ diameter for sewers usually with less than $25 \mathrm{~mm}$ settlement.

However, many subway station tunnels produce movement over $50 \mathrm{~mm}$ settlement because of excavation of the large section tunnels involved. For example, a doubledeck-three-arch station tunnel construction often measures 
TABle 4: Preliminary assessment of the effect of ground movement on buried pipeline (Attewell et al. [3]).

\begin{tabular}{|c|c|c|}
\hline $\begin{array}{l}\text { Maximum surface } \\
\text { settlement }(\mathrm{mm})\end{array}$ & $\begin{array}{c}\text { Brittle materials (gray iron, asbestos cement, and } \\
\text { clayware) }\end{array}$ & $\begin{array}{c}\text { Ductile materials (steel, ductile iron, uPVC, and } \\
\text { polyethylene) }\end{array}$ \\
\hline$W_{\max } \leq 10$ & $\begin{array}{r}\text { Pipe stress increase is not significant compared with } \\
\text { seasonal }\end{array}$ & $\begin{array}{l}\text { er causes of stress such as installation, traffic load, and } \\
\text { ovement }\end{array}$ \\
\hline$W_{\max }>10$ & The effects of movement should be accessed in detail & 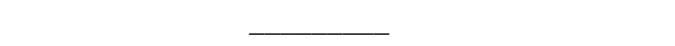 \\
\hline$W_{\max }>25$ & $\begin{array}{c}\text { Significant stress increase virtually certain; possible } \\
\text { failure of small-diameter pipes }\end{array}$ & \\
\hline$W_{\max }>50$ & Possible failure of large-diameter pipes & $\begin{array}{l}\text { Significant stress increases likely; the effects of } \\
\text { movement should be accessed in detail }\end{array}$ \\
\hline
\end{tabular}

a total of approximately $25 \mathrm{~m}$ in width and $16 \mathrm{~m}$ in height. Thus, detailed consideration should be given to the effect of this settlement on buried cast iron or steel pipelines because these types of pipelines usually remain in place during the subway station tunnel construction.

From the measured ground surface settlements listed in Table 3, it is clear that, for pipelines parallel to tunnel centreline, the steel pipeline with a diameter of $1400 \mathrm{~mm}$, and cover depth of $2.26 \mathrm{~m}$, the allowable surface settlement is at least $65.5 \mathrm{~mm}$; for the cast iron pipeline with a diameter of $300 \mathrm{~mm}$ and cover depth of $2 \mathrm{~m}$, the allowable surface settlement is at least $44.6 \mathrm{~mm}$; for the steel pipeline with a diameter of $800 \mathrm{~mm}$ and cover depth of $2.55 \mathrm{~m}$, the allowable surface settlement is at least $35.1 \mathrm{~mm}$; for the steel pipeline with a diameter of $400 \mathrm{~mm}$ and cover depth of $1.41 \mathrm{~m}$, the allowable surface settlement is at least $46 \mathrm{~mm}$; for the steel pipeline with a diameter of $500 \mathrm{~mm}$ and cover depth of $1.41 \mathrm{~m}$, the allowable surface settlement is at least $44.6 \mathrm{~mm}$. These in situ measured results provide references for similar pipelines under similar conditions.

\section{Conclusions}

Tunnel excavation inevitably produces significant disturbances to the surrounding environments; thus, the tunnelinduced influence on adjacent buried pipelines is of considerable importance for geotechnical practice. Based on analysis of the in situ measured settlements of the pipelines and the above ground surface at the Huangzhuang station, the conclusions that can be drawn are as follows:

(1) For the tunnels excavated using the PBA method, the settlement development of the buried pipelines in the close proximity can be divided into four stages. During stages 1 and 3, the increase in the pipeline settlement is not fast, whereas sharp increases occur in stages 2 and 4 . Protective measures, if applicable and necessary, should be taken over the periods of stages 2 and 4 . On the contrary, the PBA method is a multidrift sequence excavation scheme. The scheme can be adjusted to reduce the influence on the pipelines in the close proximity. For example, changing the excavation size of the top drifts can help control the pipeline settlements.
(2) For large-diameter buried steel or cast iron pipelines, separation most likely occurs from the underlying soil. In this case, monitoring the settlement of the surface above the pipeline can be used instead of monitoring the buried pipeline settlement itself. The measured results can be used to conservatively estimate the pipeline safety during construction, thereby ensuring a highly efficient and low-cost solution.

(3) In the case of a buried pipeline separating from the underlying soil, the overground surface settlement can be taken as the empirical criterion to judge pipeline safety. The large-diameter steel or cast iron pipelines, such as the monitored pipelines in the paper, can suffer a ground surface settlement of over $50 \mathrm{~mm}$ without influencing the pipeline operation and safety.

Although ground surface settlement, as the criterion to evaluate pipeline safety, is easy to implement in practice, more accumulated field-observed surface settlements are absolutely necessary for buried pipelines of different ages, materials, diameters, and buried depth.

\section{Data Availability}

The data used to support the findings of this study are included within the article.

\section{Conflicts of Interest}

The authors declare that they have no conflicts of interest regarding the publication of this paper.

\section{Acknowledgments}

The support from the Chinese National Natural Science Foundation (no. 50978017) is acknowledged.

\section{References}

[1] Q. Fang, D. Zhang, and L. N. Y. Wong, "Shallow tunnelling method (STM) for subway station construction in soft ground," Tunnelling and Underground Space Technology, vol. 29, no. 5, pp. 10-30, 2012. 
[2] X. Liu, Y. Liu, W. Qu, and Y. Tu, "Internal force calculation and supporting parameters sensitivity analysis of side piles in the subway station excavated by Pile-Beam-Arch method," Tunnelling and Underground Space Technology, vol. 56, no. 6, pp. 186-201, 2016.

[3] P. B. Attewell, J. Yeates, and A. R. Selby, Soil Movements Induced by Tunnelling and Their Effects on Pipelines and Structures, Blackie and Son Ltd., London, UK, 1986.

[4] A. Bracegirdle, R. J. Mair, R. J. Nyren, and R. N. Taylor, "A methodology for evaluating potential damage to cast iron pipes induced by tunneling," Geotechnical Aspects of Underground Construction in Soft Ground, pp. 659-664, Balkema, Rotterdam, Netherlands, 1996.

[5] Y. Wang, J. Shi, and C. W. W. Ng, "Numerical modeling of tunneling effect on buried pipelines," Canadian Geotechnical Journal, vol. 48, no. 7, pp. 1125-1137, 2011.

[6] J. Yu, C. Zhang, and M. Huang, "Soil-pipe interaction due to tunnelling: assessment of Winkler modulus for underground pipelines," Computers and Geotechnics, vol. 50, no. 5, pp. 17-28, 2013.

[7] E. S. Ieronymaki and A. J. Whittle, "Pipeline response to ground deformations induced by tunneling," in Proceedings of the ASCE Geotechnical Frontiers, pp. 566-575, Orlando, FL, USA, 2017.

[8] A. Klar, A. M. Marshall, K. Soga, and R. J. Mair, "Tunneling effects on jointed pipelines," Canadian Geotechnical Journal, vol. 45, no. 1, pp. 131-139, 2008.

[9] A. Klar, T. E. B. Vorster, K. Soga, and R. J. Mair, "Soil-pipe interaction due to tunnelling: comparison between Winkler and elastic continuum solutions," Géotechnique, vol. 55, no. 6, pp. 461-466, 2005.

[10] A. Klar, T. E. Vorster, K. Soga, and R. J. Mair, "Elastoplastic solution for soil-pipe-tunnel interaction," Journal of Geotechnical and Geoenvironmental Engineering, vol. 133, no. 7, pp. 782-792, 2007.

[11] T. E. Vorster, A. Klar, K. Soga, and R. J. Mair, "Estimating the effects of tunneling on existing pipelines," Journal of Geotechnical and Geoenvironmental Engineering, vol. 131, no. 11, pp. 1399-1410, 2005.

[12] T. E. B. Vorster, R. J. Mair, K. Soga, and A. Klar, "Centrifuge modeling of the effect of tunneling on buried pipelines: mechanisms observed," in Proceedings of the 5th International Symposium TC28 Geotechnical Aspects of Underground Construction in Soft Ground, pp. 131-136, Amsterdam, The Netherlands, 2005.

[13] A. M. Marshall, A. Klar, and R. J. Mair, “Tunneling beneath buried pipes: view of soil strain and its effect on pipeline behavior," Journal of Geotechnical and Geoenvironmental Engineering, vol. 136, no. 12, pp. 1664-1672, 2010.

[14] J. Shi, Y. Wang, and C. W. W. Ng, "Centrifuge modelling of three-dimensional tunnelling effects on buried pipeline," in Proceedings of the 32th Geotechnical Divisions' Annual Seminar, HKIE, Hong Kong, pp. 337-342, 2012.

[15] J. Shi, Y. Wang, and C. W. W. Ng, "Three-Dimensional centrifuge modeling of ground and pipeline response to tunnel excavation," Journal of Geotechnical and Geoenvironmental Engineering, vol. 142, no. 11, Article ID 04016054, 2016.

[16] A. Hunter, "Effect of trenchless technologies on existing iron pipelines," Proceedings of the Institution of Civil Engineers Geotechnical Engineering, vol. 158, no. 3, pp. 159-167, 2005.

[17] Y. Wang, Q. Wang, and K. Y. Zhang, "An analytical model for pipe-soil-tunneling interaction," Procedia Engineering, vol. 14, pp. 3127-3135, 2011.
[18] C. Zhang, J. Yu, and M. Huang, "Effects of tunnelling on existing pipelines in layered soils," Computers and Geotechnics, vol. 43, no. 6, pp. 12-25, 2012.

[19] Z. Zhang, M. Huang, and M. Zhang, "Deformation analysis of tunnel excavation below existing pipelines in multi-layered soils based on displacement controlled coupling numerical method," International Journal for Numerical and Analytical Methods in Geomechanics, vol. 36, no. 11, pp. 1440-1460, 2012.

[20] Z. Zhang and M. Zhang, "Mechanical effects of tunneling on adjacent pipelines based on Galerkin solution and layered transfer matrix solution," Soils and Foundations, vol. 53, no. 4, pp. 557-568, 2013.

[21] B. P. Wham, C. Argyrou, and T. D. O'Rourke, "Jointed pipeline response to tunneling-induced ground deformation," Canadian Geotechnical Journal, vol. 53, no. 11, pp. 1794-1806, 2016.

[22] J. Shi, Y. Wang, and C. W. W. Ng, "Numerical parametric study of tunneling-induced joint rotation angle in jointed pipelines," Canadian Geotechnical Journal, vol. 53, no. 12, pp. 2058-2071, 2016.

[23] J. Shi, X. Zhang, L. Chen, and L. Chen, "Numerical investigation of pipeline responses to tunneling-induced ground settlements in clay," Soil Mechanics and Foundation Engineering, vol. 54, no. 5, pp. 303-309, 2017.

[24] O. V. Trifonov and V. P. Cherniy, "A semi-analytical approach to a nonlinear stress-strain analysis of buried steel pipelines crossing active faults," Soil Dynamics and Earthquake Engineering, vol. 30, no. 11, pp. 1298-1308, 2010.

[25] D. K. Karamitros, G. D. Bouckovalas, G. P. Kouretzis, and V. Gkesouli, "An analytical method for strength verification of buried steel pipelines at normal fault crossings," Soil Dynamics and Earthquake Engineering, vol. 31, no. 11, pp. 1452-1464, 2011.

[26] G. P. Kouretzis, D. K. Karamitros, and S. W. Sloan, “Analysis of buried pipelines subjected to ground surface settlement and heave," Canadian Geotechnical Journal, vol. 52, no. 8, pp. 1058-1071, 2015.

[27] M. Saiyar, P. Ni, W. A. Take, and I. D. Moore, "Response of pipelines of differing flexural stiffness to normal faulting," Géotechnique, vol. 66, no. 4, pp. 275-286, 2016.

[28] P. Ni and S. Mangalathu, "Simplified evaluation of pipe strains crossing a normal fault through the dissipated energy method," Engineering Structures, vol. 167, no. 7, pp. 393-406, 2018.

[29] P. Ni, I. D. Moore, and W. A. Take, "Numerical modeling of normal fault-pipeline interaction and comparison with centrifuge tests," Soil Dynamics and Earthquake Engineering, vol. 105, pp. 127-138, 2018.

[30] S. Ma, Y. Shao, Y. Liu, J. Jiang, and X. Fan, "Responses of pipeline to side-by-side twin tunnelling at different depths: 3D centrifuge tests and numerical modelling," Tunnelling and Underground Space Technology, vol. 66, no. 6, pp. 157-173, 2017.

[31] M. Son, "Simple parametric analysis of the response of buried pipelines to micro-tunneling-induced ground settlements," Journal of the Korean Geoenvironmental Society, vol. 15, no. 11, pp. 29-42, 2014.

[32] L. Zhang, X. Wu, Y. Qin, M. J. Skibniewski, and W. Liu, "Towards a fuzzy bayesian network based approach for safety risk analysis of tunnel-induced pipeline damage," Risk Analysis, vol. 36, no. 2, pp. 278-301, 2015.

[33] X. Liu, Y. Liu, Z. Yang, and C. He, "Numerical analysis on the mechanical performance of supporting structures and ground settlement characteristics in construction process of subway 
station built by pile-beam-arch method," KSCE Journal of Civil Engineering, vol. 21, no. 5, pp. 1690-1705, 2017.

[34] T. D. O’Rourke and C. H. Trautmann, "Buried pipeline response to tunnel ground movements," in Proceedings of the Europipe 82 Conference, pp. 9-15, Basel, Switzerland, 1982. 